\title{
Warming rays in cluster cool cores
}

\author{
S. Colafrancesco ${ }^{1,2}$ and P. Marchegiani ${ }^{2,3}$
}

\author{
1 ASI-ASDC c/o ESRIN, via G. Galilei snc, 00040 Frascati, Italy \\ e-mail: colafrancesco@.asdc.asi.it \\ 2 INAF - Osservatorio Astronomico di Roma via Frascati 33, 00040 Monteporzio, Italy \\ e-mail: cola@mporzio.astro.it \\ 3 Dipartimento di Fisica, Università di Roma La Sapienza, P.le A. Moro 2, Roma, Italy \\ e-mail: marchegiani@mporzio.astro.it
}

Received 7 August 2007 / Accepted 7 January 2008

\section{ABSTRACT}

\begin{abstract}
Context. Cosmic rays are confined in the atmospheres of galaxy clusters and, therefore, they can play a crucial role in the heating of their cool cores.

Aims. We discuss here the thermal and non-thermal features of a model of cosmic ray heating of cluster cores that can provide a solution to the cooling-flow problems. To this aim, we generalize a model originally proposed by Colafrancesco, Dar \& DeRujula (2004) and we show that our model predicts specific correlations between the thermal and non-thermal properties of galaxy clusters and enables various observational tests.

Methods. The model reproduces the observed temperature distribution in clusters by using an energy balance condition in which the X-ray energy emitted by clusters is supplied, in a quasi-steady state, by the hadronic cosmic rays, which act as "warming rays" (WRs). The temperature profile of the intracluster (IC) gas is strictly correlated with the pressure distribution of the WRs and, consequently, with the non-thermal emission (radio, hard X-ray and gamma-ray) induced by the interaction of the WRs with the IC gas and the IC magnetic field.

Results. The temperature distribution of the IC gas in both cool-core and non cool-core clusters is successfully predicted from the measured IC plasma density distribution. Under this contraint, the WR model is also able to reproduce the thermal and non-thermal pressure distribution in clusters, as well as their radial entropy distribution, as shown by the analysis of three clusters studied in detail: Perseus, A2199 and Hydra. The WR model provides other observable features of galaxy clusters: a correlation of the pressure ratio (WRs to thermal IC gas) with the inner cluster temperature $\left(P_{\mathrm{WR}} / P_{\mathrm{th}}\right) \sim\left(k T_{\mathrm{inner}}\right)^{-2 / 3}$, a correlation of the gamma-ray luminosity with the inner cluster temperature $L_{\gamma} \sim\left(k T_{\text {inner }}\right)^{4 / 3}$, a substantial number of cool-core clusters observable with the GLAST-LAT experiment, a surface brightness of radio halos in cool-core clusters that recovers the observed one, a hard X-ray ICS emission from cool-core clusters that is systematically lower than the observed limits and yet observable with the next generation high-sensitivity and spatial resolution HXR experiments like Simbol-X.

Conclusions. The specific theoretical properties and the multi-frequency distribution of the e.m. signals predicted in the WR model render it quite different from the other models so far proposed for the heating of clusters' cool-cores. Such differences make it possible to prove or disprove our model as an explanation for the cooling-flow problems on the basis of multi-frequency observations of galaxy clusters.
\end{abstract}

Key words. cosmology: theory - galaxies: clusters: general - ISM: cosmic rays

\section{Introduction}

Theoretical description of the intra-cluster (IC) plasma in the central regions of many clusters predicts that it is cooler than in their outskirts, because it radiates X-rays at such a rate that the plasma cooling time is much shorter than the age of the cluster. However, such rapid cooling is not observed and the central-temperature depression in cluster cores is not as deep as expected on the basis of the plasma's cooling rate: the observed central temperature $T_{\text {inner }}$ settles, in fact, at a fraction $\sim 1 / 3-1 / 2$ of the outer temperature $T_{\text {outer }}$ (see, e.g. McNamara 1997; Peterson et al. 2003; Piffaretti et al. 2005; Donahue et al. 2005; see also Bregman 2004, for a review). The lack of cooling gas with $T<T_{\text {inner }}$ in cluster cores is also supported by the absence of line emission corresponding to gas below $T_{\text {inner}}$, as shown by high spatial resolution imaging with Chandra (e.g. McNamara et al. 2000; Fabian 2000; Blanton et al. 2001; Allen et al. 2001; Lewis et al. 2002; Blanton et al. 2003) and high spectral resolution measurements with the XMM-Newton RGS instrument (Peterson et al. 2001; Tamura et al. 2001;
Kaastra et al. 2001; Kahn et al. 2002; Peterson et al. 2002) and the EPIC instrument (Böhringer et al. 2001, 2002; Molendi \& Pizzolato 2001; Matsushita et al. 2002). These are referred to here as the cooling-flow (CF) problems.

It has been widely recognized that some form of heating is necessary to quench the cooling flow and form a warm-core in the IC gas structure. Available sources of heating in cluster cores are provided by AGN jets and lobes, pressure waves, buoyant bubbles and cavities, intra-cluster shock waves, leptonic and hadronic cosmic-rays.

The presence of a central radio source with jet and lobes, the mechanical heating provided by pressure waves produced by the jetted AGNs, the formation of buoyant bubbles filled with energetic plasma, the thermal conduction from the hot outer layers of the clusters, may certainly alleviate the CF problems (see e.g., Böhringer et al. 2002; Churazov et al. 2002; Ruszkowski \& Begelman 2002; Fabian 2004; Voit \& Donahue 2005; Brüggen \& Kaiser 2002; Ruszkowski et al. 2004; Vernaleo \& Reynolds 2006; Reynolds et al. 2005). 
Some difficulties with solutions along these lines, however, might occur: a first difficulty is that not all cooling flow clusters contain a powerful radio source at their center (e.g., the cluster RXJ0820.9+0752, Bayer-Kim et al. 2002; and the clusters A1650 and A2244, Donahue et al. 2005), nor a large amount of spherically distributed buoyant bubbles out to the cluster's core boundary. Another difficulty is that the bremsstrahlung cooling rate is proportional to $n_{\mathrm{e}}^{2} T^{1 / 2}$, with $n_{\mathrm{e}}$ the plasma's electron number density ${ }^{1}$ and $T$ its local temperature: a suitable conduction and/or heating and pressure-building mechanism must somehow adapt itself to this behaviour, particularly as a function of $n_{\mathrm{e}}(r)$, which varies by orders of magnitude along the clusters' radii. Other difficulties stand out with the spatial distribution of the energy deposited by AGN jets and cavities: on one hand, the energy released from AGN jets and cavities seems to be sufficient to quench cooling flow and increase the central gas entropy (e.g., Sijacki \& Springel 2006); on the other hand, it is not yet clear if such energy can be properly distributed to reproduce the observed cool-core temperature profiles (see, e.g., discussion by Vernaleo \& Reynolds 2005, 2006; Heinz et al. 2006; Vernaleo \& Reynolds 2007). Small duty cicles and a higher fraction of AGNs in cluster cores (see Bird et al. 2007) might however weaken the difficulties related to the AGN heating scenarios. In addition, there seems to be a small AGN activity in groups (Dwarakanath \& Nath 2006).

Sound waves and shocks do not seem to be efficient in quenching cooling flows (see, e.g., Fujita \& Suzuki 2006).

Irrespective of the nature of the heating source, it seems that a general property of the heating agent - which derives from the smoothness and from the similarity of the heating distribution required to quench cooling in several clusters - is that they must be spatially distributed in the whole cluster core. In fact, it has been shown (Fabian \& Sanders 2006; Fabian et al. 2006; Sanders \& Fabian 2007) that the non-thermal pressure in the Perseus cluster (one of the best studied clusters with cool cores and mini radiohalos) is quite similar to (but slightly steeper than) the thermal one.

In this context, it has been shown that a CR population in clusters that is radially distributed similarly to the radial profile of the IC gas, is able to recover the observed radial structure of the IC gas temperature in several clusters (Colafrancesco et al. 2004). The CRs in this last theory are not confined to the disk of the Galaxy, but permeate a much larger halo, being produced by "cannonballs" travelling for kiloparsecs in the interstellar medium of galaxies, decelerating by knocking out its constituents, which are thereby accelerated to become $\mathrm{CRs}^{2}$ (see, e.g., DeRújula 2004, for a review, and references therein).

Beyond the debate on the cannonball picture (see, e.g., Hillas 2006; Dar \& DeRújula 2006) and its relevance for the CR origin, we stress here that any mechanism that produces radial distributions of WRs similar to the profile of the IC gas can provide quenching of cooling flows by the action of WR heating.

In this paper we consider and justify, on the basis of a consistent picture of CR diffusion in cluster atmospheres (see Appendix A), that the WR number density $n_{\mathrm{WR}}(r)$ can be, in fact, distributed with a radial profile that is similar to that of the IC gas, and follows the general form $n_{\mathrm{WR}} \sim n_{\mathrm{e}}^{\alpha}$, i.e. it is proportional to a power $\alpha$ of the IC gas density $n_{\mathrm{e}}(r)$. The total amount of WRs in our model and the slope $\alpha$ of the $n_{\mathrm{WR}}(r)$ profile are

\footnotetext{
1 Hydrogen in the plasma is fully ionized, and we are using here $n_{\mathrm{p}} \approx n_{\mathrm{e}}$.

2 When referring to CRs in CF clusters, we use the expressions "CRs" and "WRs" as synonyms.
}

left as free parameters that subsequently will be constrained by fitting the temperature profile of the IC gas in each specific cluster core. The resulting CR density profile will be further tested against the entropy and pressure profiles in the cluster cores, the spectrum and surface brightness profile of the cluster radio halos and the future observations in the hard X-ray and gamma-ray energy bands.

We will first show that the presence of WRs in cluster cores are able to heat the IC gas and reproduce their radial temperature profiles. We will then start from this constraint to discuss the consequences that such a population of WR hadrons (protons), produced and stored in the atmospheres of galaxy clusters, has for the gamma-ray emission, for the hard X-ray (HXR) emission and for the presence of diffuse radio emission of both cool-core and non cool-core clusters. We will finally show that it is possible to use these predictions, in the framework of a multi-wavelength observational strategy, to set constraints on the amount and spatial distribution of WRs in clusters, and therefore distinguish between the WR model discussed here and other models for the onset of warm cores in galaxy clusters.

The structure of the paper is the following: in Sect. 2 we delineate the theoretical framework that is able to model the temperature structure of the cluster cool cores and determine the main parameters (i.e. the radial distribution and the density of WRs) that fit the cluster temperature profiles. In Sect. 3 we will also discuss the density and pressure distribution of WRs in the clusters' atmospheres that is consistent with the temperature profile. In Sect. 4 we will apply our procedure to a sample of ten well studied clusters. These clusters have been chosen according to the following criteria: i) four clusters that have cool cores and diffuse radio emission (these clusters certainly have a population of CRs in their centers); ii) 2 clusters with radio halos (i.e. with CRs in their atmospheres) but without cool cores, in order to compare their properties with the radio-active, cool-core clusters; iii) four clusters with cool cores but with no evident central diffuse radio emission in order to compare these non radio-active clusters with the previous ones. In Sect. 4 we also study the thermal and non-thermal pressure structure of the selected clusters and we will show that the pressure ratio $P_{\mathrm{WR}} / P_{\text {th }}$ found at the cluster center correlates with the central gas temperature $T_{\text {inner }}$ providing evidence for the basic regulation mechanism induced by WRs. We will discuss the predictions of our model for some specific clusters (like Perseus, A2199 and Hydra) for which detailed thermal and non-thermal pressure information is available as well as information on their radial entropy distribution. In Sect. 5 we will discuss the expected gamma-ray luminosity and flux of the cluster sample considered here that is correlated with the cool-core temperature structure predicted in our model. In Sects. 6 and 7 we will present analogous considerations for the expected radio and HXR emissions that are constrained by the cluster temperature structure. We will discuss the differences between our model and other scenarios for cool-core heating in Sect. 8 and we will give our main conclusions in Sect. 9.

Throughout the paper, we use a flat, vacuum-dominated cosmological model with $\Omega_{\mathrm{m}}=0.3, \Omega_{\Lambda}=0.7$ and $h=0.7$.

\section{Modelling cluster cool cores}

The virial expectation for the temperature of an un-magnetized plasma in a spherical cluster with mass $M(r)$ within a radius $r$ is:

$$
\begin{aligned}
k T(r) & \simeq \frac{G m_{\mathrm{p}} M(r)}{3 r} \\
& \simeq 2 \mathrm{keV}\left[\frac{M(r)}{10^{13} M_{\odot}}\right]\left[\frac{r}{100 \mathrm{kpc}}\right]^{-1},
\end{aligned}
$$


(see, e.g. Colafrancesco et al. 2004). The observed outer temperature $T_{\text {outer }}$ of the IC plasma - at large radii where the cooling time should be longer than the cluster's age - is roughly compatible with the virial expectation. In the inner regions of many clusters the temperature is somewhat smaller $\left(\sim 1 / 2-1 / 3\right.$ of $\left.T_{\text {outer }}\right)$ than that obtained form Eq. (1), but significantly larger than the cooling rate would imply (see, e.g., Peterson et al. 2001). To reproduce the observed temperature profile from the inner cool region to the outer hot region, we will consider a population of WRs spatially distributed in the cluster atmosphere (as obtained by solving the transport equation, see Appendix A) that efficiently heats the IC gas.

The effect of the "warming rays" on a cluster's temperature distribution will be computed under the following simplifying assumptions:

i) spherical symmetry;

ii) the radial distribution of the WR density is approximately proportional to a power $\alpha$ of the thermal plasma density, i.e. $n_{\mathrm{WR}} \propto n_{\mathrm{e}}^{\alpha}$, with $\alpha$ and the normalization constant being free parameters. In Appendix A we show that the effect of a simple model of cosmic ray diffusion in cluster atmospheres can provide such a kind of radial distribution, for quite general CR source terms;

iii) the temperature of the plasma in its "initial state" (as the cluster is born) is approximated by a radius-independent constant value;

iv) cooling by X-ray emission and heating and pressurebuilding by WRs are the dominant evolutionary agents. Flows and cluster ageing are relatively unimportant.

Given this framework, the rate per unit volume at which the IC plasma loses energy by thermal bremsstrahlung emission of $\mathrm{X}$-rays is:

$$
\begin{aligned}
\left.\frac{\mathrm{d} \epsilon}{\mathrm{d} t}\right|_{\mathrm{X}} & =a\left[n_{\mathrm{e}}(r)\right]^{2} \sqrt{T(r, t)} \\
a= & \sqrt{\frac{2^{11} \pi^{3}}{3^{3}}} \frac{e^{6} \sqrt{m_{\mathrm{e}}}}{h m_{\mathrm{e}}^{2} c^{3}} \bar{G} \bar{z} \\
& \sim 4.8 \times 10^{-24} \bar{z} \frac{1}{\sqrt{\mathrm{keV}}} \frac{\mathrm{erg} \mathrm{cm}}{\mathrm{s}}
\end{aligned}
$$

where $\bar{z}$ is the average charge of the IC plasma (we have approximated here the Gaunt factor $\bar{G}$ by unity). We write the IC gas number density as $n_{\mathrm{e}}(r)=n_{\mathrm{e} 0} \cdot g(r)$, where the function $g(r)$ contains all the radial dependence of the IC gas density and is obtained from X-ray observations of each specific cluster. The rate per unit volume at which the WRs deposit energy in the IC plasma is proportional to $n_{\mathrm{WR}}(r) \cdot n_{\mathrm{e}}(r)$ and it is therefore:

$$
\left.\frac{\mathrm{d} \epsilon}{\mathrm{d} t}\right|_{\mathrm{WR}}=b \cdot\left[n_{\mathrm{WR}}(r)\right] \cdot\left[n_{\mathrm{e}}(r)\right]=b \cdot n_{\mathrm{WR}, 0} \cdot n_{\mathrm{e} 0} \cdot[g(r)]^{1+\alpha},
$$

since we have assumed that the WR number density writes as $n_{\mathrm{WR}}=n_{\mathrm{WR}, 0} \cdot[g(r)]^{\alpha}$, i.e. proportional to $\left[n_{\mathrm{e}}(r)\right]^{\alpha}$.

The evolution of the temperature anywhere in the IC plasma satisfies the energy-conservation relation:

$$
3 k n_{\mathrm{e}}(r) \frac{\mathrm{d} T(r, t)}{\mathrm{d} t}=\left.\frac{\mathrm{d} \epsilon}{\mathrm{d} t}\right|_{\mathrm{WR}}-\left.\frac{\mathrm{d} \epsilon}{\mathrm{d} t}\right|_{\mathrm{X}},
$$

with the initial condition $T(r, 0)=T_{\mathrm{i}}$. We can rewrite Eq. (4) as:

$$
-\frac{\mathrm{d} T(r, t)}{\sqrt{T}-(b / a)\left[n_{\mathrm{WR}}(r) / n_{\mathrm{e}}(r)\right]}=\frac{a n_{\mathrm{e}}(r)}{3 k} \mathrm{~d} t
$$

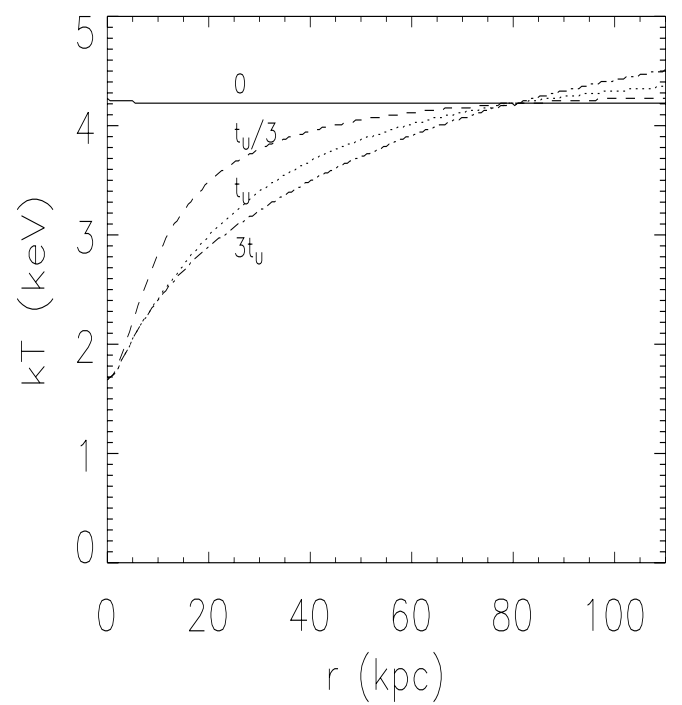

Fig. 1. The evolution of the IC gas temperature profile predicted for a cluster similar to A2199 is shown at times $t=0$ (solid), $t_{\mathrm{U}} / 3$ (dashed), $t_{\mathrm{U}}$ (dotted) and $3 t_{\mathrm{U}}$ (dot-dashed), with $t_{\mathrm{U}}$ being the age of the Universe. We assume an initial temperature of $4.2 \mathrm{keV}$ and a density profile equal to that observed in A2199. Values $\alpha=0.83$ and $n_{\mathrm{WR}, 0}=2.3 \times 10^{-3} \mathrm{~cm}^{-3}$ are assumed for this specific calculation.

and integrate, to obtain:

$$
\begin{array}{r}
\ln \left[\frac{\sqrt{T_{\mathrm{i}} / T_{\mathrm{f}}}-1}{\sqrt{T(r, t) / T_{\mathrm{f}}}-1}\right]-\frac{\sqrt{\frac{T(r, t)}{T_{\mathrm{f}}}}+\sqrt{\frac{T_{\mathrm{i}}}{T_{\mathrm{f}}}}=}{} \\
\frac{b n_{\mathrm{WR}}(r) n_{\mathrm{e}}(r)}{6 n_{\mathrm{e}}(r) k T_{\mathrm{f}}}\left(t-t_{\mathrm{i}}\right),
\end{array}
$$

where

$k T_{\mathrm{f}} \equiv\left[(b / a)\left(n_{\mathrm{WR}, 0} / n_{\mathrm{e} 0}\right) g(r)^{\alpha-1}\right]^{2}$

is the steady temperature first reached in the central region of the cluster at small radii $r$, where the density $n_{\mathrm{e}}(r)$ is high, and at an asymptotically late time in the periphery, where the density $n_{\mathrm{e}}(r)$ is vanishingly small. The r.h.s. of Eq. (6) has been written in a way that shows how the evolution of $T(r, t)$ is determined by the ratio between the WR deposition rate, $b n_{\mathrm{WR}}(r) n_{\mathrm{e}}(r)$, and the thermal energy per unit volume, $n_{\mathrm{e}}(r) k T_{\mathrm{f}}$, in the inner region where the cluster's temperature is $T_{\mathrm{f}}$.

The temperature evolution for a cluster similar to A2199 (we assume here the same IC gas density profile of A2199 and its outer temperature) as described by Eq. (6), is shown in Fig. 1 at epoch $t=0, t_{\mathrm{U}} / 3, t_{\mathrm{U}}$ and $3 t_{\mathrm{U}}$, where $t_{\mathrm{U}}$ is the current age of the Universe. This figure shows that, in our model, a cluster starts as an isothermal system at high redshifts (i.e., $t \approx 0$ ) and then a warm-core develops with time under the influence of cooling and heating effects balancing each other in order to provide a stationary configuration at each epoch. We note that the specific choice of the initial epoch $t_{i}$ is not very important in the solution of Eq. (6), because the epoch $t$ (redshift $z$ ) at which the cluster is observed is much larger (smaller) than the initial epoch $t_{i}$ (redshift $z_{i} \gg z$ ). We have nonetheless calculated the temperature evolution by assuming different values of the initial redshift, $50 \leq z\left(t_{i}\right) \leq 200$, and we found that no appreciable difference appears in the final state of the temperature radial distribution.

In cool-core clusters the central density is high enough for the equilibrium between X-ray cooling and WR heating to have been reached at the cluster's center in a time of the order of its 
current age. In such a case, $T_{\mathrm{f}} \simeq T_{\text {inner, }}$, i.e. the presently observed inner-cluster temperature. For all the clusters we study, the cooling time in the outer parts of the cluster is much longer than $t_{\mathrm{U}}$, so that $T_{\mathrm{i}} \simeq T_{\text {outer }}$. According to the value of $T_{i}$, of the IC gas density $n_{\mathrm{e}}$ and of the time $t$, Eq. (6) predicts that some clusters have reached this stage, while others have not.

The procedure described here is used to fit the temperature profile of clusters with and without cool cores. Given the bremsstrahlung energy loss rate, the temperature radial profile of each cluster depends on the density and on the spectrum $n_{\mathrm{WR}}(E, r)$ of WRs in clusters.

\section{Warming Rays in cool core clusters}

We consider a spectrum of relativistic WRs (protons) as given by:

$N_{\mathrm{WR}}(E, r)=N_{\mathrm{WR}, 0} E^{-s}[g(r)]^{\alpha}$,

with $s$ typically in the range $\approx 2.3-3.3$ (see, e.g., Marchegiani et al. 2007), and we use $s=2.7$ as a reference value, hereafter, for energies larger than $E_{\min }=m_{\mathrm{p}} c^{2} \cdot\left[1+3.4 \times 10^{-5}(\mathrm{kT} / \mathrm{keV})\right]$ (see, e.g. Furlanetto \& Loeb 2002). The value of $E_{\max }$ is usually very large and is therefore irrelevant in our calculations for spectral indices $s>2$.

The quantity $N_{\mathrm{WR}, 0}$ can be derived by assuming that the WR heating rate $b \cdot n_{\mathrm{WR}} \cdot n_{\mathrm{e}}$ provided by the energy lost by the WRs when they interact with the IC medium, recovers the IC gas temperature profile through Eq. (6).

The fundamental processes by which the warming rays can heat the IC gas are mainly Coulomb and hadronic interactions with the plasma nuclei and magneto hydro-dynamical (MHD) waves excited by CR proton anisotropies.

For moderately relativistic CR nuclei with a charge $z$, velocity $v=\beta c$ and Lorentz factor $\gamma$, the Coulomb energy-loss rate is:

$$
-\frac{\mathrm{d} E}{\mathrm{~d} t} \approx K z^{2} Z^{2} \frac{1}{\beta}\left[\ln \frac{2 m_{\mathrm{e}} c^{2} \beta^{2} \gamma^{2}}{I_{\mathrm{p}}}-\beta^{2}\right],
$$

where $Z^{2}$ is the (suitably averaged) squared charge of the plasma's nuclei, $K=4 \pi n_{\mathrm{e}} r_{\mathrm{e}}^{2} m_{\mathrm{e}} c^{3}$ and $r_{\mathrm{e}}=e^{2} / m_{\mathrm{e}} c^{2} \simeq 2.82 \mathrm{fm}$. Here $I_{\mathrm{p}}=\hbar \omega_{\mathrm{p}}$, with $\omega_{\mathrm{p}}=\left[4 \pi n_{\mathrm{e}} e^{2} / m_{\mathrm{e}}\right]^{1 / 2}$ the plasma frequency $^{3}$. The heating rate given in Eq. (9), for typical plasma densities near the center of clusters $\left(n_{\mathrm{e}} \sim 10^{-2} \mathrm{~cm}^{-3}\right)$, is $\sim 4$ times larger than for neutral hydrogen. For protons $(z=1)$ with a typical WR energy $(\gamma=2)$ and for a plasma consisting of $\sim 0.93 \%$ $\mathrm{H}, \sim 0.07 \% \mathrm{He}$ (by number) and traces of heavier elements, one finds $Z^{2} \sim 1.14$ and $\mathrm{d} E / \mathrm{d} t \approx 10^{-17} \mathrm{GeV} / \mathrm{s}$, for the quoted electron number density.

Hadronic collisions between WRs and plasma nuclei constitute an energy-loss mechanism that is approximately as efficient as that given in Eq. (9). Here we consider the collisions of WR protons with ambient protons, the consideration of protonnucleon and nucleon-nucleon collisions being far too cumbersome to be justified by the small admixtures of nuclei heavier than protons in the WR and IC constituencies. For values

\footnotetext{
${ }^{3}$ It has been noticed by Colafrancesco et al. (2004) that a highly ionized plasma is much more efficient than non-ionized gas in slowing down the CR nuclei. Energy losses via Coulomb collisions with neutral atoms become significant only for energy transfers larger than their ionization potential $I$. The corresponding small impact parameters imply relatively small cross sections. For the IC plasma, the role of $I$ is played by the much smaller quantity $I_{\mathrm{p}}$.
}

$\gamma>2$ of the incident proton, the $p p$ cross section is $\sigma_{p p} \sim$ $4 \times 10^{-26} \mathrm{~cm}^{2}$, and it is dominantly inelastic. In practically all of these collisions, the incident proton survives unscathed, as the "leading" (most energetic) final-state particle, carrying on average $\approx 70 \%$ of the incident proton's energy. Thus, a proton's energy-loss rate by hadronic collisions in a hydrogenic plasma is

$$
-\frac{\mathrm{d} E}{\mathrm{~d} t} \sim 0.7 n_{\mathrm{e}} \sigma_{p p} c E
$$

(Mannheim \& Schlickeiser 1994), that reads $\mathrm{d} E / \mathrm{d} t \sim 1.6 \times$ $10^{-17} \mathrm{GeV} / \mathrm{s}$, for our reference value of $n_{\mathrm{e}}$ and $\gamma=2$. This is very close to the Coulomb energy loss previously computed for the same reference parameters.

An additional process that has been discussed in the literature is the effect of the MHD waves excited by proton anisotropy, which might occur as a relativistic, charged particle stream faster than the local Alfven speed (see, e.g. Skilling 1971); these waves can be dissipated efficiently in the background ICM via Landau damping and other dissipative mechanisms (see, e.g., Foote \& Kulsrud 1979). The effect of this mechanism in cluster atmospheres has been studied in detail by Loewenstein et al. (1991): under the assumptions that the wave velocity is large enough to heat the IC gas significantly, and that the Coulomb and hadronic heating can be neglected, these authors found that the thermal pressure becomes overwhelmed by the CR proton pressure. Therefore, they concluded that such a CR heating mechanism alone is not able to stabilize the cooling flows.

Following the previous considerations, we assume therefore that the Coulomb and hadronic heating are the dominant sources and we search for the amount and spatial distribution of WRs required to produce the temperature structure observed in a sample of clusters with cool cores (i.e. where heating is required) and radio halos (i.e., where CRs are required). In Sect. 4.3 we will check our results by comparing the non-thermal and thermal pressures predicted in our model with those observed in the same clusters.

The total WR loss rate is given by the sum of Eqs. (9) and (10)

$\left(\frac{\mathrm{d} E}{\mathrm{~d} t}\right)_{\text {tot }} \simeq n_{\mathrm{e}}\left(A_{\text {coul }}+A_{\text {hadr }}\right)$

with

$A_{\text {coul }}=4 \pi r_{\mathrm{e}}^{2} m_{\mathrm{e}} c^{3} z^{2} Z^{2} \frac{1}{\beta}\left[\ln \frac{2 m_{\mathrm{e}} c^{2} \beta^{2} \Gamma^{2}}{I_{\mathrm{p}}}-\beta^{2}\right]$

$A_{\text {hadr }} \simeq 0.7 \sigma_{p p} c E$

and is approximately proportional to $n_{\mathrm{e}}$ (by neglecting the logarithmic dependence on $n_{\mathrm{e}}$ contained in $\omega_{\mathrm{p}}$ in Eq. (9)).

The value of $b$ (see Eq. (3)) is found by convolving the energy loss rate with the WR spectrum and equating with the warming rate

$\left.\frac{\mathrm{d} \epsilon}{\mathrm{d} t}\right|_{\mathrm{WR}} \equiv \int_{E_{\min }}^{E_{\max }} N_{\mathrm{WR}}(E, r)\left(\frac{\mathrm{d} E}{\mathrm{~d} t}\right)_{\mathrm{tot}} \mathrm{d} E$,

which writes as

$b \cdot n_{\mathrm{WR}}(r) \cdot n_{\mathrm{e}}(r) \equiv N_{\mathrm{WR}, 0} g^{\alpha}(r) n_{\mathrm{e}}(r) I_{\mathrm{A}}$,

where

$I_{\mathrm{A}} \equiv \int_{E_{\min }}^{E_{\max }} E^{-s}\left(A_{\text {coul }}+A_{\text {hadr }}\right) \mathrm{d} E$ 
Table 1. The list of clusters analyzed. Redshift $z$, inner observed temperature $T_{\text {inner }}$ and outer observed temperature $T_{\text {outer }}$ are given. The last two columns show which cluster has a radio halo $(\mathrm{CC}=$ cool-core; $\mathrm{NCC}=$ non cool-core; $\mathrm{RH}=$ radio halo) and the references of the data we used for the fit.

\begin{tabular}{cccccc}
\hline \hline Cluster & $z$ & $\begin{array}{c}T_{\text {inner }} \\
\mathrm{keV}\end{array}$ & $\begin{array}{c}T_{\text {outer }} \\
\mathrm{keV}\end{array}$ & Notes & Ref. \\
\hline A262 & 0.0162 & 0.95 & 2.4 & $\mathrm{CC}$ & {$[1]$} \\
A2199 & 0.030 & 1.6 & 4.2 & $\mathrm{CC}$ RH & {$[2]$} \\
A133 & 0.057 & 2.05 & 4.61 & $\mathrm{CC}$ Relic & {$[1]$} \\
Perseus & 0.0179 & 3.04 & 6.7 & $\mathrm{CC}$ RH & {$[3]$} \\
Hydra & 0.0539 & 3.1 & 4.0 & $\mathrm{CC}$ & {$[4]$} \\
A1795 & 0.0625 & 3.5 & 6.5 & $\mathrm{CC}$ & {$[1]$} \\
A2390 & 0.2304 & 4.89 & 9.81 & $\mathrm{CC} ; \mathrm{RH}$ & {$[1]$} \\
RX J1347.5-1145 & 0.451 & 7.04 & 19.4 & $\mathrm{CC} ; \mathrm{RH}$ & {$[5]$} \\
\hline Coma & 0.023 & 8.2 & 8.2 & $\mathrm{NCC}$ RH & {$[6]$} \\
A2163 & 0.203 & 14.6 & 14.6 & $\mathrm{NCC}$ RH & {$[7]$} \\
\hline
\end{tabular}

[1] Vikhlinin et al. (2005); [2] Johnstone et al. (2002); [3] Churazov et al. (2003); [4] David et al. (2001); [5] Allen et al. (2002); [6] Briel et al. (1992); [7] Elbaz et al. (1995).

Since the WR density at the cluster's center is given by

$n_{\mathrm{WR}, 0}=\int_{E_{\min }}^{E_{\max }} \mathrm{d} E N_{\mathrm{WR}, 0} E^{-s}$,

we obtain

$b=\frac{I_{\mathrm{A}}}{\int_{E_{\min }}^{E_{\max }} \mathrm{d} E E^{-s}}$.

Inserting the previous expression of $b$ in Eq. (6) we obtain the value of $n_{\mathrm{WR}, 0}$ required to fit the temperature profile, and in turn the pressure of the WRs as

$P_{\mathrm{WR}}(r)=\frac{1}{3} m_{\mathrm{p}} c \int_{\gamma_{\min }}^{\gamma_{\max }} N_{\mathrm{WR}}(\gamma, r) \beta \gamma v(\gamma) \mathrm{d} \gamma$,

where $\gamma=E_{\mathrm{p}} /\left(m_{\mathrm{p}} c^{2}\right)$.

\section{The effect of WRs in cluster cores}

\subsection{Temperature profiles}

The solution of Eq. (6) provides a fit to the cluster's temperature profile by adjusting the free parameters $\alpha$ and $n_{\mathrm{WR}, 0}$. We have applied this analysis to ten well studied clusters, eight of which are cool-core (CC) clusters and two of which are non cool-core (NCC) clusters (see Table 1). Specifically, we considered 4 clusters with cool-cores which have central radio emission (usually of the class of mini radio-halos): these are A2199, Perseus, A2390 and RX J1347.5-1145. For comparison, we also considered two well known non cool-core clusters with radio halos (Coma and A2163) and some of the best studied clusters with cool-cores but no evident diffuse radio emission, i.e. A262, A133 (which has a relic radio emission feature), Hydra and A1795.

For each cluster in Table 1, we found the values of $\alpha$ and $n_{\mathrm{WR}, 0}$ that best fit the observed temperature profile, by assuming that initially (i.e. at high $z$ when the cluster formed) each cluster was isothermal with temperature $T_{i}$ equal to the outer observed temperature, and that the evolution of the temperature proceeds according to Eq. (6) and finishes at the redshift $z$ at which each cluster is observed.

The WR density $n_{\mathrm{WR}}(r)$ found through this procedure is the one required to heat the IC gas at the level shown by the temperature profile by using Eq. (6).
We notice that the WR density in cool-core clusters is required to be flatter (i.e. with values $\alpha<1$ ) than the IC gas density to provide the heating rate necessary to quench the gas cooling and reproduce the cluster's temperature profile. On the other hand, clusters without cool-cores require $\alpha \approx 1$ because they have not yet developed gas cooling conditions (see discussion at the end of Sect. 2).

\subsection{Pressure and density of WRs}

The ratio of the central WR pressure $P_{\mathrm{WR}}$ to the IC gas thermal pressure $P_{\text {th }}$ is found by using Eq. (18) and the expression $P_{\text {th }}=2 n_{\mathrm{e}}(r) k T(r)$ (by including the contribution of both thermal protons and electrons). The value of the best-fit central pressure ratio $P_{\mathrm{WR}} / P_{\text {th }}$ found for each cluster considered in this study is reported in Table 2.

The results of our fitting procedure to the cluster temperature profiles indicate that there is a strong correlation between the inner cluster temperature $T_{\text {inner }}$ and the pressure ratio $P_{\mathrm{WR}} / P_{\text {th }}$ at the cluster center (see Fig. 2). This correlation can be well represented with a power-law function $P_{\mathrm{WR}} / P_{\text {th }} \propto\left(k T_{\text {inner }}\right)^{\delta}$ with $\delta \approx-2 / 3$.

Such a correlation is due to the fact that in clusters with cooler inner temperature, a high IC gas density triggers fast gas cooling; therefore, a stronger warming action (compared to the thermal energy) is required to quench the cooling and to adjust the temperature profile to the observed one by the energy-conservation relation in Eq. (6). The central pressure ratio $P_{\mathrm{WR}} / P_{\text {th }}$ that is required in order to equilibrate gas cooling and then settle the IC gas at the inner temperature value $T_{\text {inner }}$ is obtained from Eq. (7) and reads

$$
\begin{aligned}
P_{\mathrm{WR}} / P_{\mathrm{th}} & =\frac{1}{2 \sqrt{k T_{\text {inner }}}}\left(\frac{a}{b}\right) \frac{P_{\mathrm{WR}}}{n_{\mathrm{WR}, 0}} \\
& =\frac{1}{2 \sqrt{k T_{\text {inner }}}}\left(\frac{a}{b}\right)\left\langle\varepsilon_{\mathrm{WR}}\right\rangle .
\end{aligned}
$$

The correlation $P_{\mathrm{WR}} / P_{\text {th }} \propto\left(k T_{\text {inner }}\right)^{-2 / 3}$ is explained by the combined temperature dependence of the term $\propto\left(k T_{\text {inner }}\right)^{-1 / 2}$ and of the quantity $\left(\frac{a}{b}\right)\left\langle\varepsilon_{\mathrm{WR}}\right\rangle \sim\left(k T_{\text {inner }}\right)^{-1 / 6}$, which depends on $T_{\text {inner }}$ through the lower limit of integration $E_{\text {min }}\left(T_{\text {inner }}\right)$ in the expressions of $b$ and $\left\langle\varepsilon_{\mathrm{WR}}\right\rangle$ (see Fig. 3). Note that the $T_{\text {inner }}$ dependence of $b$ and $\left\langle\varepsilon_{\mathrm{WR}}\right\rangle$ arises only from the spectral properties of WRs in our model, while the scaling with $\left(k T_{\text {inner }}\right)^{-1 / 2}$ arises from the condition set in Eq. (6).

The other correlation, which is naturally related to the previous one, is found - as expected - between the central WR density $n_{\mathrm{WR}, 0}$ and the value of $k T_{\text {inner }}$ (see Fig. 4). In fact, from Eq. (7) we obtain that

$k T_{\mathrm{f}}=\left(\frac{b}{a} \cdot \frac{n_{\mathrm{WR}, 0}}{n_{\mathrm{e} 0}}\right)^{2}$

for $r \rightarrow 0$. Since the WRs are normalized to obtain $T_{\mathrm{f}} \sim T_{\text {inner }}$, we can write

$n_{\mathrm{WR}, 0} \sim a \sqrt{k T_{\mathrm{inner}}} \cdot n_{\mathrm{e} 0} \cdot \frac{I_{\mathrm{E}}}{I_{\mathrm{A}}}$

where $I_{\mathrm{E}}=\int_{E_{\min }}^{E_{\max }} \mathrm{d} E E^{-s}$ and $I_{\mathrm{A}}$ is defined in Eq. (15). Then the ratio $I_{\mathrm{E}} / I_{\mathrm{A}}$ depends on $E_{\min }$ and, as a consequence, on $k T_{\text {inner }}$; we found that for our range of temperatures this dependence is 
Table 2. Column 1: cluster name; Col. 2: $\alpha$; Col. 3: $n_{\mathrm{WR}, 0}$ (in units of $\mathrm{cm}^{-3}$ ); Col. 4: the pressure ratio $P_{\mathrm{WR}} / P_{\mathrm{th}}$ at the cluster center; Col. 5: the gamma-ray flux $F_{\gamma}$ in units of $\mathrm{cm}^{-2} \mathrm{~s}^{-1}$; Col. 6: the gamma-ray luminosity $L_{\gamma}$ in units of $\mathrm{erg} \mathrm{s}^{-1}$; (the gamma-ray luminosity and flux are integrated in the 0.1-10 GeV band.) Col. 7: the HXR flux $F_{\mathrm{HXR}}$ in units of $\mathrm{erg} \mathrm{cm}^{-2} \mathrm{~s}^{-1}$ integrated in the $10-50 \mathrm{keV}$ band.

\begin{tabular}{ccccccc}
\hline \hline Cluster & $\alpha$ & $\begin{array}{c}n_{\mathrm{WR}, 0} \\
\mathrm{~cm}^{-3}\end{array}$ & $P_{\mathrm{WR}} / P_{\mathrm{th}}$ & $\begin{array}{c}F_{\gamma} \\
\mathrm{cm}^{-2} \mathrm{~s}^{-1}\end{array}$ & $\begin{array}{c}L_{\gamma} \\
\mathrm{erg} \mathrm{s}^{-1}\end{array}$ & $\begin{array}{c}F_{\mathrm{HXR}} \\
\mathrm{erg} \mathrm{cm}^{-2} \mathrm{~s}^{-1}\end{array}$ \\
\hline A262 & 0.83 & $2.20 \times 10^{-3}$ & 1.23 & $3.89 \times 10^{-9}$ & $1.43 \times 10^{42}$ & $3.87 \times 10^{-14}$ \\
A2199 & 0.83 & $2.31 \times 10^{-3}$ & 0.92 & $8.43 \times 10^{-9}$ & $1.08 \times 10^{43}$ & $3.06 \times 10^{-13}$ \\
A133 & 0.84 & $4.56 \times 10^{-4}$ & 0.77 & $7.30 \times 10^{-10}$ & $3.53 \times 10^{42}$ & $6.10 \times 10^{-15}$ \\
Perseus & 0.91 & $4.98 \times 10^{-4}$ & 0.54 & $2.20 \times 10^{-8}$ & $9.91 \times 10^{42}$ & $1.59 \times 10^{-13}$ \\
Hydra & 0.97 & $6.24 \times 10^{-4}$ & 0.57 & $3.46 \times 10^{-9}$ & $1.49 \times 10^{43}$ & $2.57 \times 10^{-14}$ \\
A1795 & 0.96 & $5.55 \times 10^{-4}$ & 0.50 & $3.17 \times 10^{-9}$ & $1.86 \times 10^{43}$ & $2.41 \times 10^{-14}$ \\
A2390 & 0.94 & $2.21 \times 10^{-4}$ & 0.41 & $1.41 \times 10^{-10}$ & $1.39 \times 10^{43}$ & $6.17 \times 10^{-16}$ \\
RX J1347.5-1145 & 0.89 & $9.20 \times 10^{-4}$ & 0.33 & $5.07 \times 10^{-10}$ & $2.37 \times 10^{44}$ & $8.66 \times 10^{-16}$ \\
Coma & 1.00 & $1.07 \times 10^{-5}$ & 0.29 & $1.65 \times 10^{-8}$ & $1.23 \times 10^{43}$ & $1.67 \times 10^{-13}$ \\
A2163 & 1.00 & $1.42 \times 10^{-5}$ & 0.21 & $1.31 \times 10^{-9}$ & $9.71 \times 10^{43}$ & $4.85 \times 10^{-15}$ \\
\hline
\end{tabular}

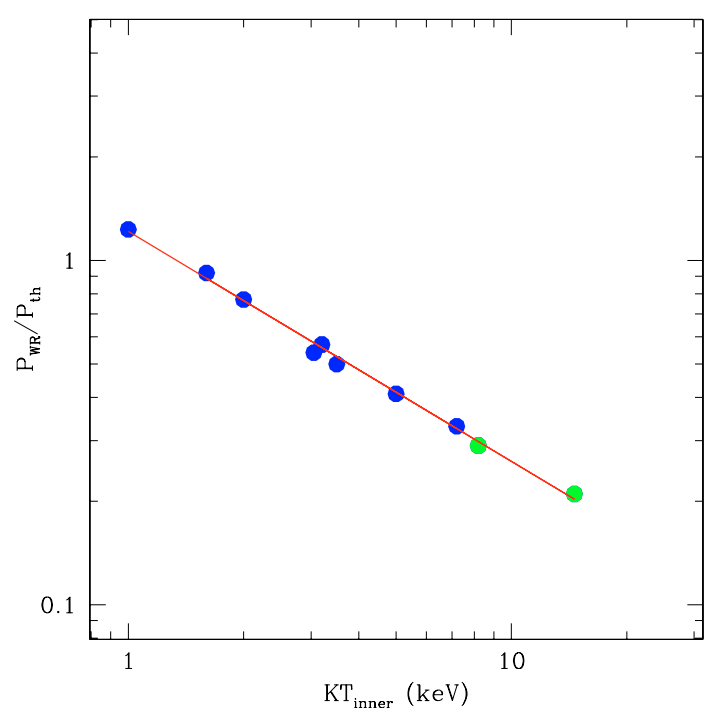

Fig. 2. The correlation of the central pressure ratio $P_{\mathrm{WR}} / P_{\mathrm{th}}$ to the inner temperature of the cool core $T_{\text {inner }}$ in $\mathrm{keV}$ is given for each cluster in Table 1. The fit $P_{\mathrm{WR}} / P_{\text {th }} \propto\left(k T_{\text {inner }}\right)^{-2 / 3}$ is shown as a solid line. Blue dots refer to cool-core clusters and green dots to non cool-core clusters.

$I_{\mathrm{E}} / I_{\mathrm{A}} \propto\left(k T_{\text {inner }}\right)^{-1.7}$. The conclusion is that

$\frac{n_{\mathrm{WR}, 0}}{n_{e 0}} \propto\left(k T_{\text {inner }}\right)^{-1.2}$,

which is confirmed by our results (see Fig. 5).

\subsection{Pressure profiles: the cases of Perseus and A2199}

We compare the pressure profiles of the (non-thermal) WRs and of the thermal components obtained in our model by fitting the IC gas temperature profile to the data available for Perseus and A2199.

For Perseus we find that a central ratio $P_{\mathrm{WR}} / P_{\text {th }} \approx 0.54$ is required in order to reproduce the inner temperature profile; such a value is consistent with that found between the non-thermal and the thermal pressures in the analysis of Sanders \& Fabian (2007). It is also remarkable that our model is able to reproduce the steeper spatial profile of the non-thermal pressure w.r.t. the thermal pressure profile found by these authors in their analysis of the central regions of Perseus (see Fig. 6). We note also that the non-thermal pressure we find in this cluster is lower than the

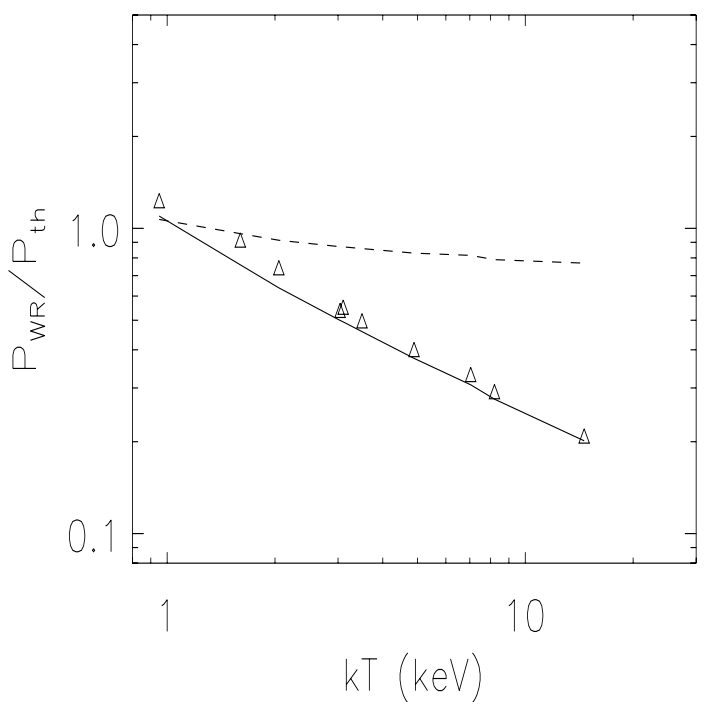

Fig. 3. The contribution of the term $\left(\frac{a}{b}\right)\left\langle\varepsilon_{\mathrm{WR}}\right\rangle$ (dashed curve) to the correlation of the pressure ratio $P_{\mathrm{WR}} / P_{\mathrm{th}}$ with the inner temperature of the cool core $T_{\text {inner }}$ is shown. The solid curve is the convolution of the terms $\left(\frac{a}{b}\right)\left\langle\varepsilon_{\mathrm{WR}}\right\rangle$ and $\left(k T_{\text {inner }}\right)^{-1 / 2}$ (see text for details).

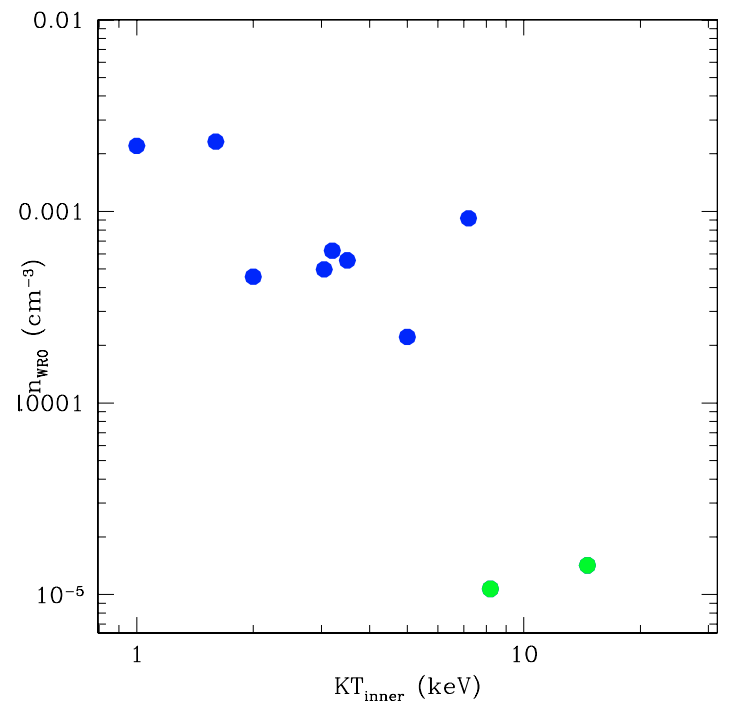

Fig. 4. The correlation of the central WR density $n_{\mathrm{WR}, 0}$ with the inner temperature of the cool core $T_{\text {inner }}$. Blue dots refer to cool-core clusters and green dots to non cool-core clusters. 


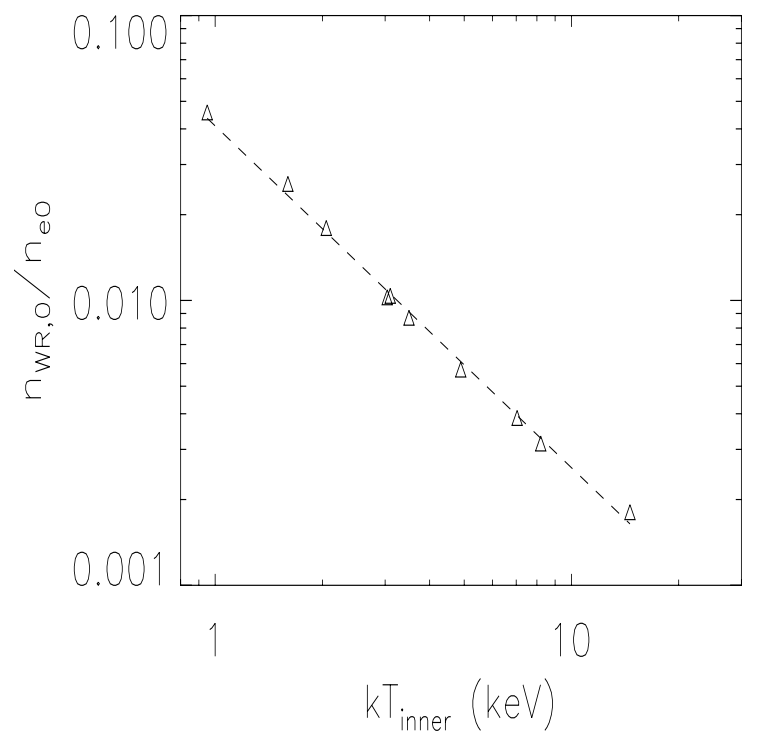

Fig. 5. The correlation of the ratio $n_{\mathrm{WR}, 0} / n_{\mathrm{e} 0}$ with the inner temperature of the cool core $T_{\text {inner. }}$. The dashed line shows the trend $n_{\mathrm{WR}, 0} / n_{\mathrm{e} 0} \propto$ $\left(k T_{\text {inner }}\right)^{-1.2}$.

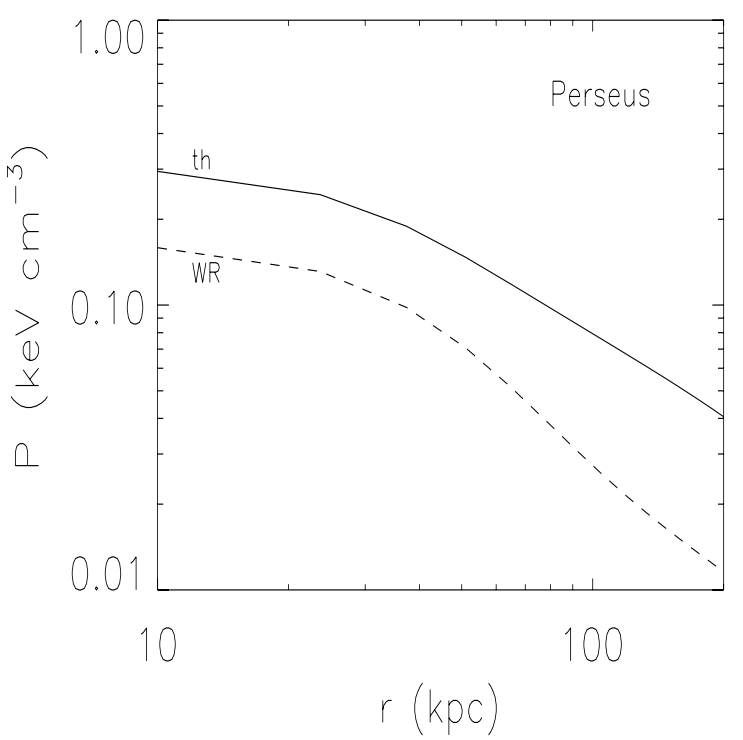

Fig. 6. The radial profile of the WR pressure (dashed curve) of Perseus compared to that of the thermal gas (solid curve). The radial profile of the WR pressure resembles the radial profile of the non-thermal pressure found by Sanders \& Fabian (2007).

thermal one: in this respect, we can conclude that our model does not have the problems shown by scenario of CR heating provided by waves excited by proton anisotropy (see Loewenstein et al. 1991). In our framework, the non-thermal pressure component found in the core of Perseus can be entirely attributed to WRs. These results bring our WR heating model to a strong consistency level with the results of the available X-ray observations (e.g. Sanders \& Fabian 2007). As a consequence of the presence of WRs in the core of Perseus cluster, we expect a non-thermal HXR emission due to ICS of the secondary electrons from the central region of Perseus: we will discuss in Sect. 7 the predictions of our model for the HXR emission in the $2-10 \mathrm{keV}$ band where the instruments on board Chandra and XMM-Netwon are sensitive.

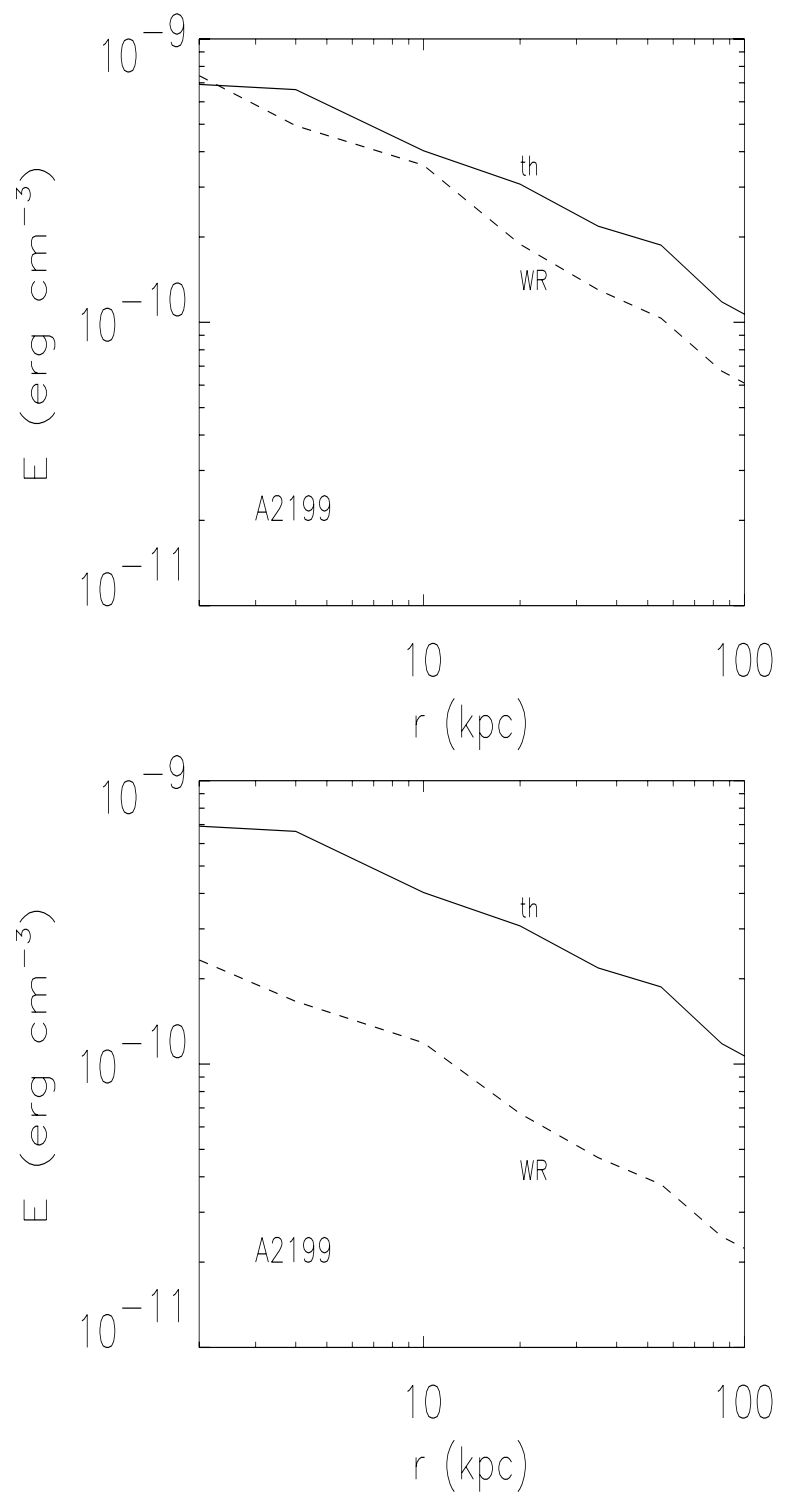

Fig. 7. The radial profile of the WR energy density of A2199 compared to that of the thermal gas. The two panels refer to i) a proton spectrum with $\gamma_{\min }=1+3.4 \times 10^{-5}(\mathrm{kT} / \mathrm{keV})$ (see, e.g., Furlanetto \& Loeb 2002); ii) a proton spectrum with $E_{\text {kin,min }}=706 \mathrm{MeV}$.

We have also studied the energy density profile of the WRs and of the thermal IC gas in A2199. The WR energy density has been evaluated as

$E_{\mathrm{WR}}(r)=m_{\mathrm{p}} c^{2} \int_{\gamma_{\min }}^{\gamma_{\max }} N_{\mathrm{WR}}(\gamma, r)(\gamma-1) \mathrm{d} \gamma$

and again depends on the WR spatial distribution that fits the cluster temperature profile. The thermal IC gas energy density is evaluated as $E_{\mathrm{th}}=3 n_{\mathrm{e}}(r) k T(r)$ (by including the contribution of both thermal protons and electrons). The comparison of the two energy densities as evaluated in our model is shown in the upper panel of Fig. 7. Our model predicts that the WR energy density at the center of A2199 is approximately equal to the IC gas thermal energy in order to fit the cluster's temperature profile. Similarly to the case of Perseus, we find a radial profile of the WR energy density that decays faster with radius than the thermal energy density.

It is possible to recover the energy density ratio of A2199 as presented in the model of Guo \& Oh (2007) by noticing 
that the minimum energy cutoff chosen by these last authors is $\approx 706 \mathrm{MeV}$, i.e. much higher than the value of $E_{\min }$ assumed in this paper (see Sect. 3). In fact, when we cut the WR spectrum at this higher value of $E_{\min }$, we recover an energy density ratio that is quite close to the value found in Guo \& Oh (2007). We verified that the residual difference in this ratio is due to the marginal effect of thermal conduction in their model.

\subsection{Entropy profiles}

The WR heating in the cluster core can be also responsible for the flattening of the IC gas entropy profile as observed in cool-core clusters. We have compared the entropy profiles predicted in our model for Perseus, A2199 and Hydra to the available data for these clusters (see e.g., Churazov et al. 2003; Caffi et al. 2004; David et al. 2001) and we found a very good agreement (see Fig. 8). This is expected because our model is able to reproduce the cluster temperature profile given the observed density profile of the cluster and therefore their combination $S=k T_{\text {inner }} / n_{\mathrm{e}}^{2 / 3}$ which is referred to as the IC gas entropy. This analysis bring further robustness to the ability of our model to explain the structure of clusters' cores in terms of a balance between WR heating and IC gas cooling.

\section{Gamma-ray emission of cool core clusters}

The distribution of WRs that heat the IC gas to recover the observed temperature profiles in their inner regions inevitably produces gamma-ray emission due to various emission mechanisms: i) $p p \rightarrow \pi^{0} \rightarrow \gamma+\gamma$; ii) inverse Compton scattering (ICS) due to secondary electrons produced by $p p \rightarrow \pi^{ \pm} \rightarrow \mathrm{e}^{ \pm}$; iii) non-thermal bremsstrahlung emission of the same secondary electrons.

From the WR spectra discussed in this paper (see Sect. 4), we calculated the gamma-ray emission produced by the decay of the $\pi^{0} \rightarrow \gamma \gamma$ produced in the interactions between relativistic protons and the thermal gas target protons (see, e.g., Blasi \& Colafrancesco 1999; Marchegiani et al. 2007, for the details of the calculations). The contribution of secondary electrons to the gamma-ray emission via ICS of CMB photons and non-thermal bremsstrahlung is negligible w.r.t. the $\pi^{0} \rightarrow \gamma \gamma$ flux (see also Marchegiani et al. 2007) and, therefore, we do not consider them in the following discussion.

Note that, at variance with previous works that considered the non-thermal (radio, HXR and gamma-ray) emission of CRs in cluster atmospheres (e.g., Colafrancesco \& Blasi 1998; Blasi \& Colafrancesco 1999; Miniati et al. 2001a,b; Pfrommer \& Ensslin 2004; Marchegiani et al. 2007; Pfrommer et al. 2007), the density of the WRs is not a free parameter in our model, because it is fixed by the procedure presented in Sect. 2 used to fit the temperature profile of the cluster. Therefore, the cluster's gamma-ray emission is determined only by this condition and it is strongly related to the density and temperature structure of the cluster's cool core.

The total gamma-ray flux and luminosity consistent with the cool-core temperature profile of the considered clusters are reported in Table 2 . The cluster gamma-ray luminosity predicted in our model shows an overall increase with the value of $T_{\text {inner }}$ even though with some scatter. The total cluster gamma-ray luminosity due to $p p \rightarrow \pi^{0} \rightarrow \gamma \gamma$ decay scales with the density of the WRs and of the IC gas according to $L_{\gamma} \propto n_{\mathrm{WR}}(r) n_{\mathrm{p}}(r) R^{3}$, and it is integrated out to the radius at which we see target thermal protons from the clusters' X-ray images. The condition
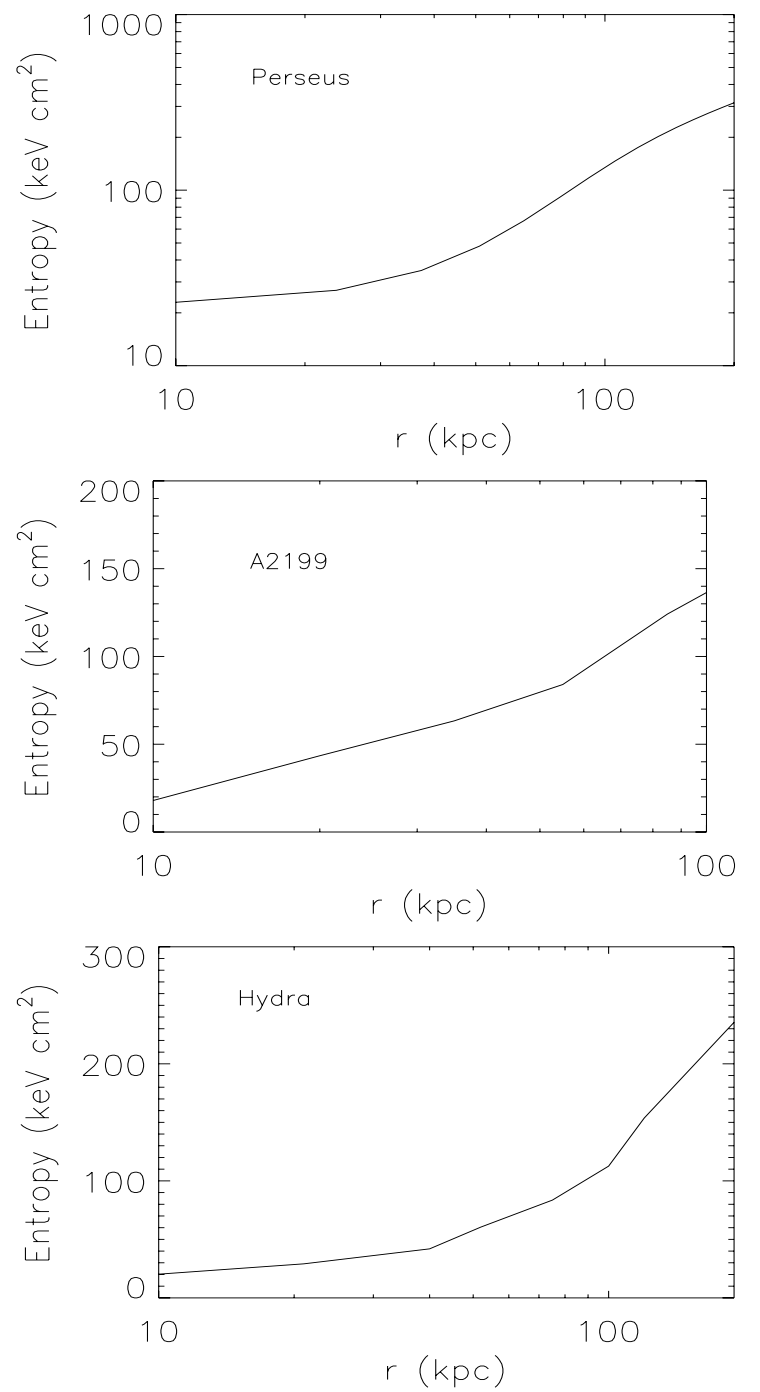

Fig. 8. The radial profile of the IC gas entropy $S$ of Perseus (upper panel), A2199 (mid panel) and Hydra (lower panel) as predicted in the WR model. The curves reproduce the observed entropy profiles of the three clusters as derived by Churazov et al. (2003) for Perseus, by Caffi et al. (2004) for A2199 and by David et al. (2001) for Hydra.

imposed by the WR heating model to fit the inner temperature profile yields a scaling $L_{\gamma} \propto \sqrt{k T_{\text {inner }}}(a / b) n_{\mathrm{e} 0}^{2} R^{3}$ which can be rewritten as $L_{\gamma} \propto \sqrt{k T_{\text {inner }}}(a / b) L_{\mathrm{X}} /(k T)^{1 / 2}$ and yields a scaling $L_{\gamma} \propto\left(k T_{\text {inner }}\right)^{\beta}(a / b)$ by using the observed $L_{\mathrm{X}} \sim T^{\beta}$ relation (see Arnaud 2005 for a review) and a value $k T_{\text {inner }} \approx(1 / 3-1 / 2) k T$. The scaling of $L_{\gamma}$ with $\left(k T_{\text {inner }}\right)^{1.3}$ that derives form the previous considerations reproduces quite well the overall increase of the gamma-ray luminosity of the ten clusters in Table 1 as a function of their inner core temperature $k T_{\text {inner. }}$.

However, a scatter in the $L_{\gamma}-T_{\text {inner }}$ relation shown in Fig. 9 is expected because the cluster gamma-ray luminosity depends on the different values of $\alpha$ and on the different values of the integration volume $\propto R^{3}$ chosen for each specific cluster. The choice of different integration volumes for the considered clusters is motivated by the different extensions of the target protons in the thermal gas to produce effective $p p \rightarrow \pi^{0} \rightarrow \gamma \gamma$ collisions, as indicated by the available X-ray data for each cluster (see Table 1). A systematic increase of $L_{\gamma}$ with $T_{\text {inner }}$ is however shown by the data and can be understood by the previous scaling-law considerations. 


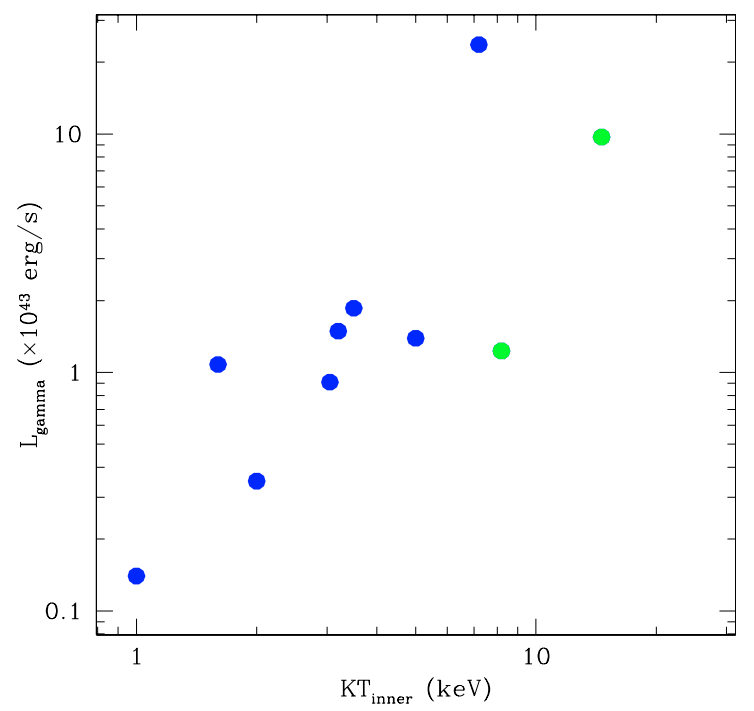

Fig. 9. The correlation of the gamma-ray luminosity $L_{\text {gamma }}$ with the inner temperature of the cool core $T_{\text {inner. }}$. Blue dots refers to cool-core clusters and green dots to non cool-core clusters.

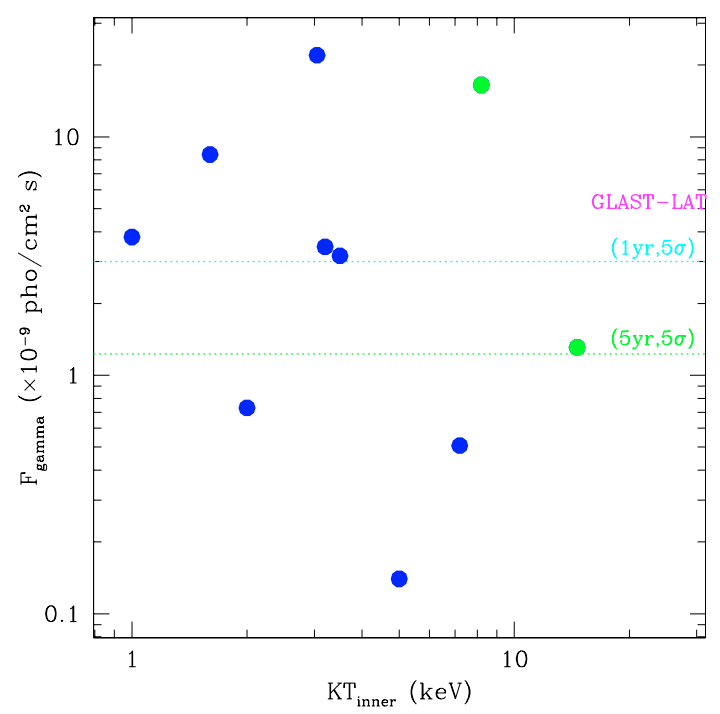

Fig. 10. The distribution of the gamma-ray flux $F_{\text {gamma }}(E>0.1 \mathrm{GeV})$ with the inner temperature of the cool core $T_{\text {inner. }}$. Blue dots refer to cool-core clusters and green dots to non cool-core clusters. We show the GLAST-LAT sensitivity for a $5 \sigma$ detection after $1 \mathrm{yr}$ (cyan line) and after $5 \mathrm{yr}$ (green line) (from Ritz 2007, on behalf of the GLAST collaboration see http://wWw-glast.slac.stanford.edu/software/IS/ glast_lat_performance.htm).

We have also calculated the gamma-ray flux expected from each cluster in Table 2 and we compared the results with the GLAST-LAT instrument sensitivity (see Fig. 10). While all the gamma-ray fluxes are lower than the available EGRET limits (Reimer et al. 2003), it is evident that 6 clusters with $F_{\gamma} \geq$ $3 \times 10^{-9}$ photons cm ${ }^{-2} \mathrm{~s}^{-1}$ (A262, A2199, Perseus, Hydra, A1795 and Coma), and 7 clusters with $F_{\gamma} \geq 1.2 \times 10^{-9}$ photons $\mathrm{cm}^{-2} \mathrm{~s}^{-1}$ (the previous clusters plus A2163) could be detected by the GLAST-LAT experiment in 1 year and 5 years at the $5 \sigma$ confidence level, respectively.

The possible gamma-ray detection of these clusters will allow us to probe in detail our model for the WR heating of the cluster cores and consequently set constraints on the parameters $n_{\mathrm{WR}, 0}$ and $\alpha$ of the WR heating model.
We stress that the gamma-ray emission evaluated here for cool-core clusters refers to the truly diffuse component arising from $p p \rightarrow \pi^{0} \rightarrow \gamma \gamma$ decay. We do not consider here the possible contribution due to the gamma-ray emission from the central engine of the radio galaxies and /or from their radio lobes. We will address more completely this issue in a forthcoming paper.

Gamma-ray emission from WRs is able to directly probe the hadronic component of their interaction with the IC gas. Nonetheless, the leptonic component of the WRs - IC gas interaction can be also efficiently probed by studying the secondary $\mathrm{e}^{ \pm}$e.m. emission: this is spread over a wide range of frequency, from radio (where the synchrotron emission dominates) to the UV, X-ray and HXRs (where the ICS emission dominates). We will discuss these components separately in the following sections.

\section{Diffuse radio emission in cool core clusters}

Diffuse radio emission is also inevitably produced by synchrotron emission of the secondary electrons produced by $p p \rightarrow$ $\pi^{ \pm} \rightarrow \mathrm{e}^{ \pm}$interacting with the intra-cluster magnetic field and it is therefore strongly related to the WR gamma-ray emission and to the temperature profile of the cluster core. From the WR spectrum and spatial distribution given in Eq. (8), we calculated the radio emission produced by the secondary electrons (we follow the procedure described in Marchegiani et al. 2007), by assuming a radial profile $B(r)$ for the magnetic field.

We apply this procedure to the clusters in Table 2 where a mini-halo is observed, i.e. A2390, Perseus, RX J1347.5-1145 and A2199 (in this last case an upper limit only is available, see Kempner \& Sarazin 2000).

In the cluster A2390, we found that the intensity of the radio emission is $2 \times 10^{-2}$ and $2 \mathrm{mJy}$ for values of the central magnetic field in the range $1.3-13 \mu \mathrm{G}$ (which are equal to $B_{\text {eq }}$ and $10 B_{\text {eq }}$, respectively, where $B_{\text {eq }}$ is the value inferred by equipartion between magnetic field and electrons energy, Govoni \& Feretti 2004), and for a radial shape of the magnetic field $B(r) \propto n_{\mathrm{e}}(r)$, respectively. The radio flux associated to the A2390 mini-halo has been estimated to be $63 \pm 3 \mathrm{mJy}$ (Bacchi et al. 2003). However, the radio source at the center of A2390 is a complex combination of the radio emission from the powerful FR-II flatspectrum cD radio galaxy and of the mini halo (Augusto et al. 2006). The substantial amount of polarization detected around the $\mathrm{cD}$ radio galaxy and in the mini halo region indicates that the radio flux attributed to the mini halo region could be substantially affected by the radio galaxy lobes, therefore suggesting a lower flux of the truly diffuse halo.

For the Perseus cluster, we show in Fig. 11 the radio brightness at $1.4 \mathrm{GHz}$ calculated for a magnetic field with central intensity of $10 \mu \mathrm{G}$ and a radial profile proportional to $n_{\mathrm{e}}^{0.5}(r)$. These assumptions are comparable with those of Pfrommer \& Enßlin (2004), who performed a calculation of the radio emission of the secondary electrons in the Perseus cluster by fitting the CR density to obtain the radio intensity. We found that, for the same magnetic field they used [i.e. $B_{0}=10 \mu \mathrm{G}$ and $B(r) \propto n_{\mathrm{e}}^{0.5}(r)$ ], the WR density needed to quench the CF to the observed temperature level reproduces very well the radio halo brightness (see Fig. 11).

For the cluster RX J1347.5-1145 we found that the shape of radio emission (see Gitti et al. 2007) cannot be reproduced by secondary electrons if a monotonic radial decrease of the magnetic field is assumed. Instead, if we assume $B_{0}=1 \mu \mathrm{G}$ and a profile of $B(r) \propto n_{\mathrm{e}}^{-0.5}(r)$, we obtain the brightness shape shown in Fig. 12. This profile is very similar to that of Gitti et al. (2007), 


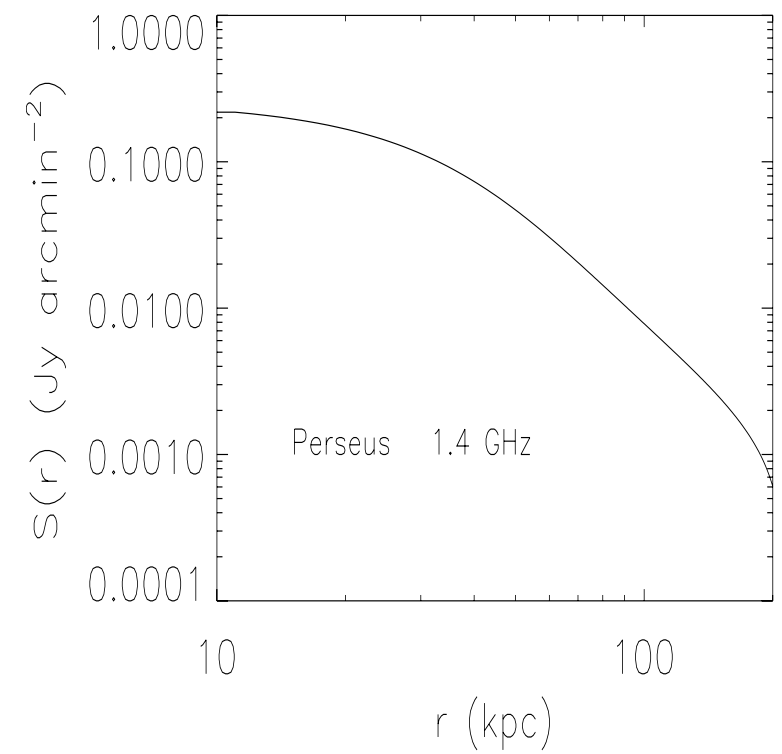

Fig. 11. Radio brightness at $1.4 \mathrm{GHz}$ emitted by secondary electrons in the Perseus cluster for $B_{0}=10 \mu \mathrm{G}$ and $B(r) \propto n_{\mathrm{e}}^{0.5}(r)$. The density and the radial shape of the WRs are those needed to stop the cooling flow at the observed temperature level.

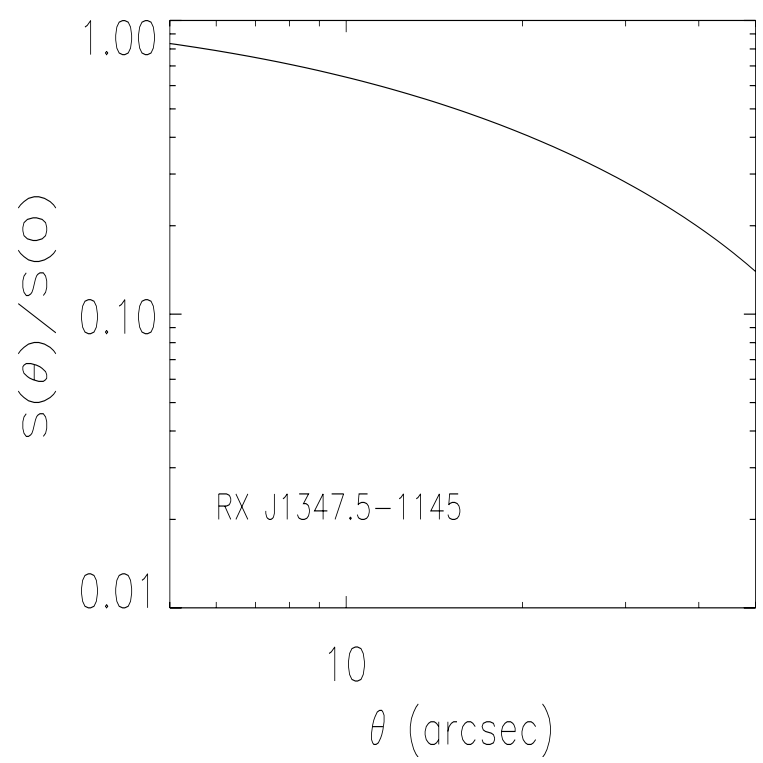

Fig. 12. Normalized radio brightness emitted by secondary electrons in the cluster RX J1347.5-1145 evaluated at $1.4 \mathrm{GHz}$ for $B_{0}=1 \mu \mathrm{G}$ and $B(r) \propto n_{\mathrm{e}}^{-0.5}(r)$. The density and the radial shape of the WRs are those needed to stop the cooling flow at the observed level.

and the total flux calculated is $33 \mathrm{mJy}$, comparable to the measured one of $\sim 25 \mathrm{mJy}$. It will be easy to recover the observed radio halo flux with a central magnetic field $\lesssim 1 \mu \mathrm{G}$.

In A2199, where we use a radial profile $B(r) \propto n_{\mathrm{e}}(r)$, we obtain a total flux of $100 \mathrm{mJy}$ for $B_{0}=5 \mu \mathrm{G}$ and $372 \mathrm{mJy}$ for $B_{0}=10 \mu \mathrm{G}$. Since the upper limit is $168 \mathrm{mJy}$ (see Kempner \& Sarazin 2000), and these values of the central magnetic field are comparable to those obtained by FR measurements in cool-core clusters, we conclude that the available radio data are compatible with our model, if the central magnetic field is not very strong, i.e. for values of $B_{0} \lesssim 7 \mu \mathrm{G}$.

For the two non cool-core clusters with radio halos considered in our analysis, we recover the results already presented in previous analyses (Marchegiani et al. 2007). Specifically, for
Coma we found $\alpha=1$, i.e. the condition $P_{\mathrm{WR}} \propto P_{\text {th }}$, and the same ratio $P_{\mathrm{WR}} / P_{\mathrm{th}} \approx 0.3$ (indicated also by the simulations of Ryu et al. 2003) for which the radio halo surface brightness can be recovered with a central magnetic field of $1.2 \mu \mathrm{G}$ (Marchegiani et al. 2007).

For A2163 we again found $\alpha=1$, i.e. the condition $P_{\mathrm{WR}} \propto$ $P_{\text {th }}$, and a ratio $P_{\mathrm{WR}} / P_{\text {th }} \approx 0.2$ for which the radio halo surface brightness can be recovered with a central magnetic field of $4.5 \mu \mathrm{G}$, a factor $\sim 2$ higher than that found by Marchegiani et al. (2007).

We note that in clusters where no diffuse radio emission has been detected, the magnetic field required by our WR model should be lower than the value required to make the radio emission from secondary electrons detectable. In fact, the synchrotron emission depends non-linearly on the magnetic field intensity and fluctuations (see discussion in Colafrancesco et al. 2005) and therefore, little or moderate variations of its amplitude can decrease the radio flux by even a large amount. Future, deeper radio observation of galaxy clusters, such as those which can be obtained with LOFAR and/or SKA, can provide further information on the intensity of the radio emission, and on the non-thermal content of galaxy cluster (see discussion in Marchegiani et al. 2007).

We note also that the WR model can provide a consistent picture for the formation of morphologically different radio sources in galaxy clusters: in fact, the diffusion processes in different clusters can produce different WR radial profiles, then minihalos or giant radio halos can be produced. In addition, the action of localized external compression (i.e. by the action of merging) can enhance the CRs density and the magnetic field intensity in external regions of the clusters, by producing the radio relics.

\section{Diffuse hard X-ray emission in cool core clusters}

The secondary electrons from WR interactions with the IC gas protons also produce radiation in the Hard X-Ray band, by Inverse Compton Scattering (ICS) of the Cosmic Microwave Background (CMB) Radiation photons.

The equilibrium spectrum of the secondary electrons depends on the density of the WRs (which is determined in our approach) and on the energy loss rates of the electrons (see, e.g., Marchegiani et al. 2007). While the ICS losses are known and are uniform all along the cluster volume, the synchrotron losses depend on $B^{2}(r)$, and therefore they are not known, in principle. However, for the clusters that host a diffuse radio emission, information on the magnetic field $B(r)$ can be inferred from the observed radio-halo features (see Sect. 6 above), while for the other clusters it is necessary to assume a magnetic field structure.

Therefore, to predict the HXR emission of clusters listed in Table 1, we adopt two different strategies: i) for the radio emitting clusters we calculate the HXR emission by using a magnetic field compatible with the observed radio emission (see Sect. 6); ii) for all the clusters we calculate the HXR emission by assuming a low magnetic field as generally indicated by the ICS interpretation of the HXR emission observed in nearby clusters. In this last case, the synchrotron losses are everywhere weaker that the ICS ones, and the density of the secondary electrons and consequently the HXR emission - has to be considered the maximum compatible with the cool-core heating by the WRs. The HXR fluxes reported in Table 2 refer to this last evaluation strategy.

In Fig. 13 the ICS emission spectrum of the secondary electrons is shown for the radio emitting clusters (A2199, Coma, 


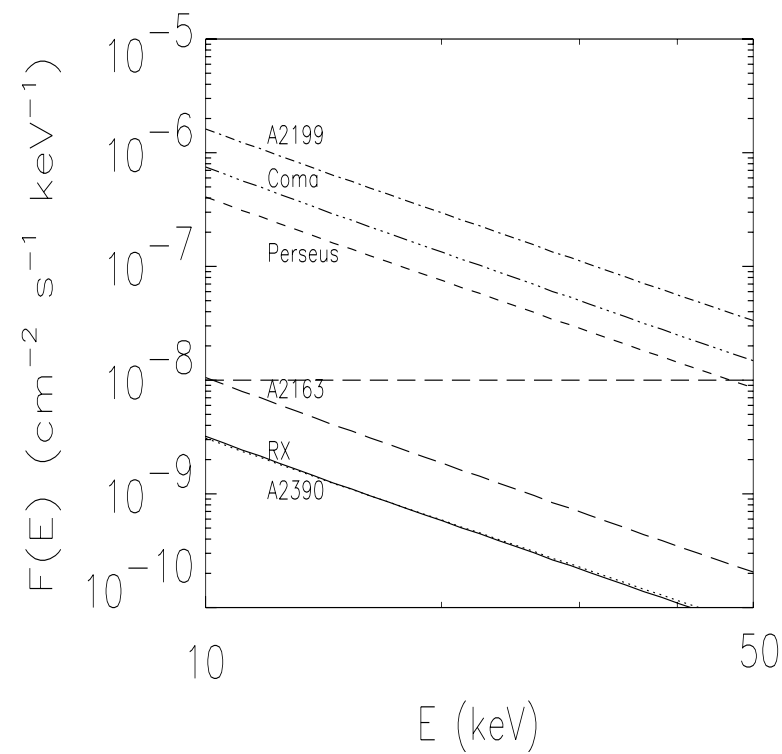

Fig. 13. ICS emission in the HXR band of the secondary electrons for the radio emitting clusters. RX stands for RX J1347.5-1145. The horizontal, long-dashed line represents the approximate sensivity of Simbol-X in the 10-50 keV range. The HXR fluxes have been computed under strategy i) (see text for details).

Perseus, A2163, A2390, RX J1347.5-1145). These fluxes, referring to the evaluation strategy i), can be compared with the expected sensitivity in the HXR band of the next coming mission Simbol-X (Ferrando et al. 2005), which is approximately $\sim 10^{-8}$ photons $\mathrm{cm}^{-2} \mathrm{~s}^{-1} \mathrm{keV}^{-1}$ in the energy band $10-50 \mathrm{keV}$. From this figure we can conclude that the clusters A2199, Coma and Perseus should be detectable by Simbol-X, if the magnetic fields in these clusters are those inferred by assuming that the radio emission is produced by secondary electrons. The HXR emission expected in the $10-50 \mathrm{keV}$ band should be clearly distinguishable from the thermal one in this energy band. On the contrary, the clusters A2163, RX J1347.5-1145 and A2390 should not be detectable by Simbol-X in this evaluation strategy (for the case of A2390 we have used a magnetic field value $\simeq 10 B_{\text {eq }}$, see discussion in Sect. 6).

In Fig. 14 (both panels), we show the HXR ICS emission of the secondary electrons in the WR model for all the clusters we considered (see Table 1), by assuming a value of the magnetic field $B_{0}=0.1 \mu \mathrm{G}$, for which energy losses are completely dominated by ICS, i.e. our evaluation strategy ii). Note that these levels of HXR emission should be considered as upper limits. From this figure, we can conclude that only RX J1347.5-1145 and A2390 should be not detectable by Simbol-X; a difficult detection is possible for A133 and A2163, while the other six clusters should be definitely detectable by Simbol-X, if the extreme conditions on the magnetic field that we have assumed hold.

The HXR fluxes predicted in the WR model are also much lower than the 20-80 keV fluxes of the nearby clusters detected with BeppoSAX (see Nevalainen et al. 2004). Specifically, we obtain for Coma a flux $F_{20-80 \mathrm{keV}}=1.6 \times 10^{-13} \mathrm{erg} \mathrm{cm}^{-2} \mathrm{~s}^{-1}$ (vs. an observed flux of $\left.F_{20-80 \mathrm{keV}}=(1.1 \pm 0.5) \times 10^{-11} \mathrm{erg} \mathrm{cm}^{-2} \mathrm{~s}^{-1}\right)$, for A2199 a flux $F_{20-80 \mathrm{keV}}=2.1 \times 10^{-13} \mathrm{erg} \mathrm{cm}^{-2} \mathrm{~s}^{-1}$ (vs. an observed flux of $\left.F_{20-80 \mathrm{keV}}=(8.4 \pm 3.9) \times 10^{-12} \mathrm{erg} \mathrm{cm}^{-2} \mathrm{~s}^{-1}\right)$ and for A2163 a flux $F_{20-80 \mathrm{keV}}=3.2 \times 10^{-15} \mathrm{erg} \mathrm{cm}^{-2} \mathrm{~s}^{-1}$ (vs. an observed flux of $\left.F_{20-80 \mathrm{keV}}=(1.7 \pm 3.1) \times 10^{-12} \mathrm{erg} \mathrm{cm}^{-2} \mathrm{~s}^{-1}\right)$. The ICS HXR fluxes yielded by the WRs in cluster cores cannot reproduce the HXR fluxes observed towards these clusters

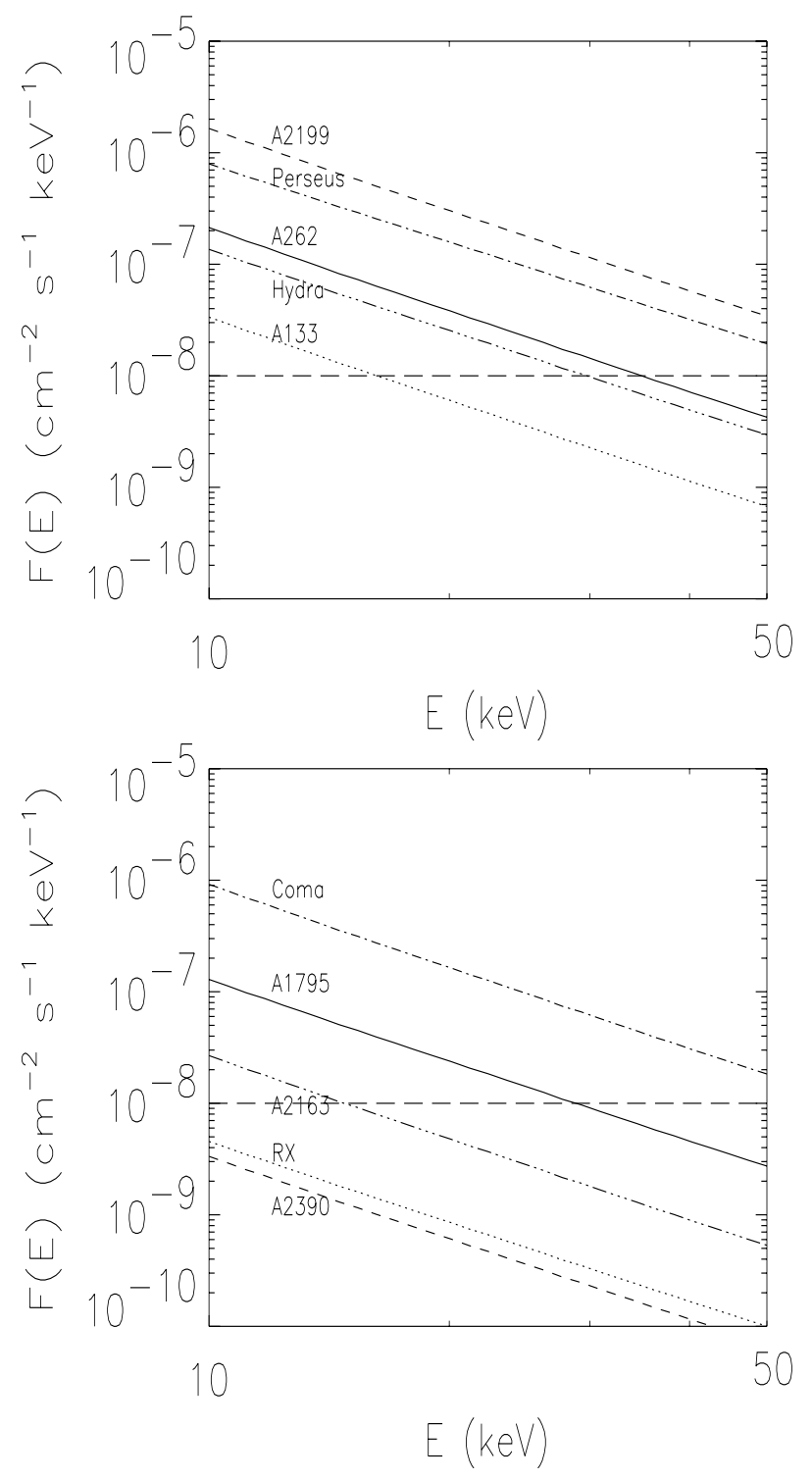

Fig. 14. ICS emission in the HXR band of the secondary electrons for all the clusters considered. For clarity, the emissions are reported in two different panels. RX stands for RX J1347.5-1145. The horizontal, long-dashed line represents the approximate sensitivity of Simbol-X in the $10-50 \mathrm{keV}$ range. The HXR fluxes have been computed here under strategy ii) (see text for details).

with BeppoSAX (see also Colafrancesco, Marchegiani \& Perola 2005 , for a general, phenomenological discussion of this point).

We also evaluated the non-thermal flux produced by ICS of the secondary electrons in the $2-10 \mathrm{keV}$ energy band intergrated in a $\sim 250$ arcsec region around the center of Perseus to be compared with the thermal bremsstrahlung emission of the IC gas in the same region of the cluster. The non-thermal ICS flux induced by WRs is a factor $\sim 10^{3}$ less than the thermal bremsstrahlung flux. This further confirms that ICS HXR fluxes yielded by the WRs in cluster cores cannot reproduce the non-thermal flux observed towards the centers of clusters.

\section{Discussion}

We have shown that a specific feature of the WR scenario for the heating of cool cores in galaxy clusters is that it is inevitably 
associated with emission features that manifest over a wide range of frequencies, from radio to gamma-rays.

This fact provides a clear test to distinguish our model from other models of cooling-flow quenching of mechanical and/or non-gravitational origin as well as from other models for the origin of non-thermal phenomena in the inner regions of galaxy clusters.

How does the WR model presented here compare with these models?

Guo \& Oh (2007) proposed a model of AGN feedback heating, in which cosmic rays produced by accretion triggered AGN activity heat the IC gas efficiently, with small dynamical perturbations on the IC gas itself. This model considers that the ICM is heated by cosmic rays (CRs), which are injected into the ICM through diffusion or the shredding of the bubbles by Rayleigh-Taylor or Kelvin-Helmholtz instabilities, with the inclusion of thermal conduction. It seems that the inclusion of CRs in this model solves the extreme fine tuning required by the conduction-suppression factor when only thermal conduction operates, and provides a more stable radial distribution of IC gas temperature that is, therefore, mainly driven by the effect of CRs which set some low value of the mass accretion rate with little additional effect of the thermal conduction (see their Fig. 3 and their discussion).

The large impact of CRs as the main heating and regulating agent of the clusters' cool-cores is quite similar to what is found in our model, because the conduction effects are small. However, our WR model requires quite substantial WR pressures at the cluster's center (that become negligible in the outer regions) that are able to recover the non-thermal pressure profile found in Perseus and help reproduce the mini radio halo surface brightness of cool-core clusters (Perseus, RX J1347.51145, A2199) with reasonable values and radial profiles of the cluster's magnetic field. The low CR pressure $\left(P_{\mathrm{CR}} \lesssim 0.1 P_{\text {th }}\right)$ and energy required by the model of Guo \& Oh (2007) in A2199 can be explained as an effect of the lower energy cutoff $\left(E_{\min } \approx 706 \mathrm{MeV}\right)$ in the CR spectrum which is much higher than our choice $\left(E_{\min }=m_{\mathrm{p}} c^{2} \times\left[1+3.4 \times 10^{-5}(k T / \mathrm{keV})\right](\right.$ see Sect. 3). We have quantitatively shown this effect in the specific case of A2199 (see Fig. 6) where we compare our WR energy density distribution with that of the CR feedback heating model of Guo \& Oh (2007). Such a difference in the energy spectrum of CRs also causes a different level of predicted gamma-ray fluxes for clusters with cool-cores, with our WR model providing higher fluxes detectable with the GLAST-LAT experiment and with the next generation HXR experiments like Simbol-X.

In particular, the Perseus cluster should be one of the best candidates to look for a HXR ICS emission feature in the $10-50 \mathrm{keV}$ range emerging from its inner core (see Figs. 13 and 14). This prediction seems quite robust since the WR distribution responsible for such ICS emission is the same one that is able to reproduce the thermal gas temperature distribution in its cool core and the mini radio halo surface brightness visible in the same spatial region. This prediction is also strengthened by the ability of the WR model to recover at the same time the thermal gas temperature radial distribution and the non-thermal (WR) pressure radial profile observed in the Perseus core (see Sanders \& Fabian 2007).

Other clusters with radio halos (e.g. Coma and the marginal detection of A2199) are also predicted to be visible by Simbol-X once we normalize our model to their temperature profile and radio-halo emission.

WR (CR) heating models are a viable alternative to mechanical heating models (e.g. Brüggen \& Kaiser 2002;
Ruszkowski et al. 2004; Vernaleo \& Reynolds 2006; Reynolds et al. 2005, McCarthy et al. 2007) in which the ICM is heated by the $p \mathrm{~d} V$ mechanical work of the expanding bubbles, viscous dissipation of emitted sound waves or mixing of the hot bubble plasma with the ICM. The detailed microphysics of how the mechanical heating processes take place has not been hammered out in detail, leaving a good deal of uncertainty; a definitive explanation for how energy is transported from the observed bubbles to the ICM in a distributed and isotropic fashion is still outstanding. The possible role of merging shocks to the heating of the IC gas in the clusters' cores is still to be quantitatively determined (see the simulations of Ryu et al. 2003).

It is clear, nonetheless, that cosmic-ray heating models also suffer from other uncertainties: the details of how WRs adjust to a density profile similar to that of the IC gas have to be addressed by specific studies that could bring further robustness to the preliminary calculations presented here (see Appendix A and Sect. 2).

It is possible to envisage various scenarios for the WR origin in terms of the activity of AGNs with small duty cycles and/or a substantial fraction of AGNs in cluster cores (see, e.g., Bird et al. 2007), or a CR origin in non-standard scenarios like cannonball models (see deRújula 2004, for review), but we do not wish to address this specific topic here. However, it seems that the WR model is somewhat less uncertain than the CR feedback heating, for which large uncertainties remain on how cosmic rays leak from the bubbles, and/or the rate at which bubbles are disrupted. Elucidating the details of bubble disruption/cosmicray diffusion would be, nonetheless, very useful in determining whether cosmic-rays or mechanical processses provide a more efficient means of transporting heat from the bubble to the ICM.

All of these issues are very important in the light of the experimental possibility to study CR physics in large-scale structures with a multi-frequency approach: the forthcoming gammaray observations of several nearby clusters with GLAST, the future HXR detection of the ICS emission from the same clusters with Simbol-X and the possible detection of nonthermal SZ effect signatures (see, e.g. Colafrancesco et al. 2003; Colafrancesco 2007, for a review) in the spectra of these clusters with SPT will help to disentangle not only the presence of cosmic rays in the cluster atmospheres, but also the details of various WR models for the heating of cluster cores.

In fact, models of mechanical heating do not show any nonthermal emission features while the WR model predicts a substantial level of non-thermal emission at various frequencies (radio, microwave, HXR and gamma) and the CR feedback heating model of Guo \& Oh (2007) predicts only marginally visible nonthermal phenomena in cool-core clusters.

The next generation radio, HXR and gamma-ray experiments will distinguish these two classes of models and will determine the amount of WRs present in galaxy clusters, provided that a good separation of the diffuse and point-like non-thermal emission will be achievable.

\section{Conclusions}

The WR heating model that we presented in this paper is able to reproduce the temperature, the pressure and the entropy structure of both cool-core and non cool-core clusters and it provides results that are consistent with all the available evidence of nonthermal phenomena emerging from the cores of clusters, e.g. diffuse radio emission in the form of mini halos and diffuse radio halos, HXR emission limits obtained with BeppoSAX, Chandra and XMM, and gamma-ray limits obtained with EGRET. 
This model provides a theoretical description of the physics of cool cores that is directly related to the presence of several observable consequences of the presence of WRs in cluster atmospheres. The main conclusions of our work are:

- the presence of WRs in cluster atmosphere provides a simple solution for the temperature, pressure and entropy profiles of the thermal IC plasma and of the non-thermal plasma observed in the inner regions of several clusters studied in this paper.

- The WR distribution produces a substantial emission of gamma-rays by their hadronic $p p \rightarrow \pi^{0} \rightarrow \gamma \gamma$ interations with the IC gas. A large fraction ( 6 clusters out of 10 considered in this study) have gamma-ray fluxes at $E>100 \mathrm{MeV}$ detectable with the GLAST-LAT experiment. The gamma-ray luminosity of the cool-core and non-cool core clusters correlates with the cluster's inner temperature, and thus provides a specific scaling behaviour of our WR model that can be verified with forthcoming GLAST observations.

- The WRs distribution can reproduce the radio emission of all the cool-core clusters with mini-halos that we studied (A2199, Perseus, RX J1347.5-1145) except for A2390 (whose mini radio-halo flux could be affected by residual radio emission from the lobes of the central $\mathrm{cD}$ radio galaxy).

- The WR distribution produces ICS emission with fluxes well below the HXR limits of A2199, Coma and A2163 provided by BeppoSAX and well below the not-thermal emission detected by Chandra and XMM in the central regions of Perseus. This means that ICS emission from WRs is not the explanation for the emission of these clusters in excess with respect to their thermal bremsstrahlung radiation.

- The distribution of WRs in clusters directly relates the thermal IC gas temperature, pressure and entropy distribution in cool-core clusters (observable in the X-ray energy band) to non-thermal diffuse emission features observable at radio (synchrotron), HXRs (ICS), gamma-rays (mainly from $\pi^{0} \rightarrow$ $\gamma \gamma$ decay) and microwaves (by Sunyaev-Zel'dovich effect). We predict that the expected levels of these non-thermal emission features in the WR model will be testable with the forthcoming experiments in the HXR band (Simbol-X), in gamma-rays (GLAST-LAT) and in microwaves (SPT). These experiments can determine the amount of WRs that are present in galaxy clusters.

- These observable predictions make the WR model testable by using a multi-frequency observational strategy that links thermal and non-thermal phenomena in clusters cores.

- The specific theoretical and observational features of the WR model render it different from other models so far proposed for the heating of cool-cores in galaxy clusters. Such peculiar differences make it possible to prove or disprove the WR model and, in general, the properties of any model proposed as an explanation for the cooling-flow problems.

Acknowledgements. We thank the referee for various suggestions.

\section{Appendix A: CR proton diffusion in cluster atmospheres}

We calculate the effect of the diffusion of CR protons (we use the same notation for CRs and WRs) in the cluster atmosphere according to the following equation:

$\frac{\partial N}{\partial t}-\nabla(D \nabla N)-\frac{\partial\left(b_{\mathrm{p}} N\right)}{\partial E}=Q_{\mathrm{p}}$ where $N\left(E_{\mathrm{p}}, r\right)$ is the proton CR density, $D\left(E_{\mathrm{p}}, r\right)$ is the diffusion coefficient, $b_{\mathrm{p}}\left(E_{\mathrm{p}}, r\right)$ is the proton energy loss term and $Q_{\mathrm{p}}\left(E_{\mathrm{p}}, r\right)$ is the source term.

Protons we are interested in here do not appreciably lose their energy on time scales of the order of the cluster's lifetime so that we can neglect the term $\partial\left(b_{\mathrm{p}} N\right) / \partial E$ in Eq. (A.1). We are interested in a quasi-stationary solution $(\partial N / \partial t=0)$, so that the diffusion equation simplifies to

$\nabla(D \nabla N)=-Q_{\mathrm{p}}$,

which, in spherical symmetry, can be written as:

$\frac{1}{r^{2}}\left[\frac{\partial}{\partial r}\left(r^{2} D \frac{\partial N}{\partial r}\right)\right]=-Q_{\mathrm{p}}$

The solution of this equation writes as:

$N(r)=\int\left[\frac{-\int \mathrm{d} r r^{2} Q_{\mathrm{p}}}{r^{2} D}\right] \mathrm{d} r$.

We search for a radial shape of the source term $Q_{\mathrm{p}}\left(E_{\mathrm{p}}, r\right)$ which, by the diffusion effect, gives a radial shape of the proton equilibrium distribution which is proportional to $[g(r)]^{\alpha}$ (this is the radial dependence of the WRs to be inserted in Eq. (4)), where $g(r)$ is the radial profile of the gas. Therefore, we write:

$\int \frac{I_{1}(r)}{r^{2} D} \mathrm{~d} r \equiv N_{0}[g(r)]^{\alpha}$

where

$I_{1}(r)=-\int_{0}^{r} \mathrm{~d} r r^{2} Q_{\mathrm{p}}(r)$

This is a general solution of Eq. (A.1) under the assumed hypothesis. Under the specific assumption that the diffusion coefficient is constant with radius, we can write

$\frac{1}{D_{0}} \int_{0}^{r} \mathrm{~d} r^{\prime} \frac{I_{1}\left(r^{\prime}\right)}{r^{\prime 2}}=N_{0}[g(r)]^{\alpha}$

By deriving both members with respect to $r$, we obtain:

$\frac{1}{D_{0}} \frac{I_{1}(r)}{r^{2}}=N_{0} \alpha[g(r)]^{\alpha-1} \frac{\mathrm{d}}{\mathrm{d} r} g(r)$,

and then

$I_{1}(r)=N_{0} D_{0} \alpha h(r)$

where

$h(r)=r^{2}[g(r)]^{\alpha-1} \frac{\mathrm{d}}{\mathrm{d} r} g(r)$.

By deriving with respect to $r$, we obtain

$r^{2} Q_{\mathrm{p}}(r)=-N_{0} D_{0} \alpha \frac{\mathrm{d}}{\mathrm{d} r} h(r)$

and then we obtain the source term that satisfies Eq. (A.3):

$Q_{\mathrm{p}}(r)=-N_{0} D_{0} \alpha \frac{1}{r^{2}} \frac{\mathrm{d}}{\mathrm{d} r} h(r)$.

This equation gives the source term $Q_{\mathrm{p}}\left(E_{\mathrm{p}}, r\right)$ which produces a radial distribution of protons proportional to $[g(r)]^{\alpha}$, for a constant diffusion coefficient $D(r) \equiv D_{0}$. Thus, we have shown that it is possible, by knowing the radial shape of the thermal gas in a cluster, to know the radial shape that the WR source term should 


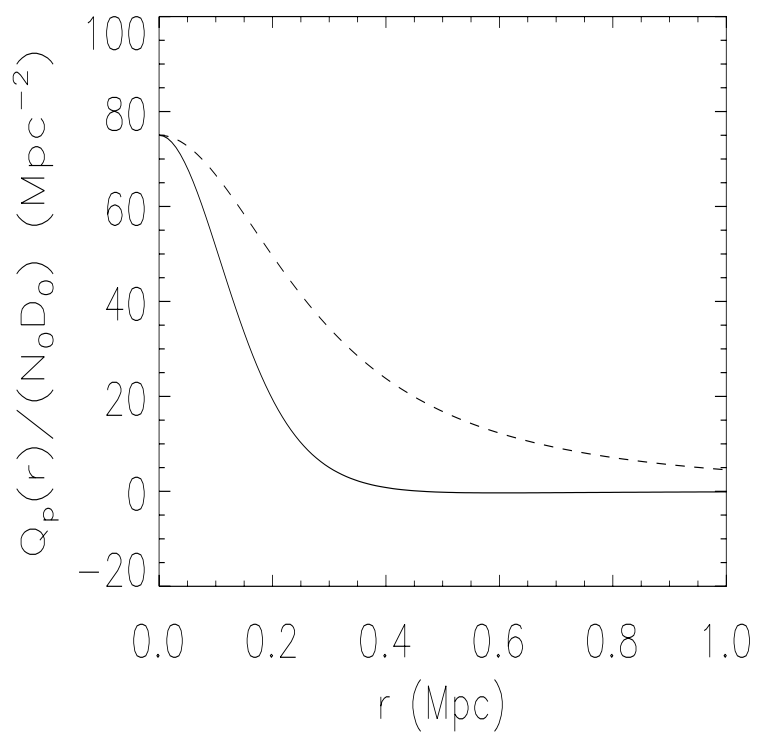

Fig. A.1. Source term [Eq. (A.12)] for the case of a cluster with the thermal profile as Coma $\left(r_{\mathrm{c}}=0.3 \mathrm{Mpc}\right.$ and $\left.q_{\mathrm{th}}=1.125\right)$ and for $\alpha=1$. The dashed line shows the thermal profile of the cluster (which, in this case, is also the resulting proton radial distribution), with an arbitrarily chosen normalization for comparison.

have in order to recover the required radial distribution of the warming rays.

In the following, we consider a solution of Eq. (A.3) for a specific case of $g(r)$, and we find the corresponding shape of the source term $Q_{\mathrm{p}}(r)$. We consider only the radial shape of the proton distribution, and we use a free normalization.

We consider a thermal IC gas profile of the form

$g(r)=\left[1+\left(\frac{r}{r_{\mathrm{c}}}\right)^{2}\right]^{-q_{\mathrm{th}}}$.

Equations (A.12) and (A.10) provide the shape of the source term $Q_{\mathrm{p}}(r)$. In Fig. A.1, we consider the case of a cluster with $r_{\mathrm{c}}=0.3 \mathrm{Mpc}$ and $q_{\mathrm{th}}=1.125$ (corresponding to the case of Coma). As we can see from this figure, the effect of the proton diffusion is to provide a broad equilibrium distribution of CRs from a narrow distribution of the proton source term.

A Gaussian distribution

$Q_{\mathrm{p}}(r) \propto \exp \left[-\left(\frac{r}{r_{\mathrm{s}}}\right)^{2}\right]$,

with a small value of $r_{\mathrm{s}}$ is able to reproduce the source term required to provide the $\mathrm{CR}$ profile $[g(r)]^{\alpha}$ with $\alpha=1$. In Fig. A.2, we compare the source term in Fig. A.1 with a Gaussian distribution as in Eq. (A.14) with $r_{\mathrm{s}}=0.17 \mathrm{Mpc}$ and two other Gaussians with $r_{\mathrm{s}}=0.10$ and $r_{\mathrm{s}}=0.05 \mathrm{Mpc}$. Then this function, with properly chosen parameters (normalization and $r_{\mathrm{s}}$ ), can be a good approximation for the required source term.

In Fig. A.3 we show the equilibrium distribution of the CR protons produced by several Gaussian source terms as in Eq. (A.14) with $r_{\mathrm{s}}=0.17,0.10$ and $0.05 \mathrm{Mpc}$, and we find that the first function is very similar to the IC gas radial distribution of a Coma-like cluster. Since this procedure is not an accurate fit, but only a qualitative comparison for an arbitrary set of parameters, we can conclude that a narrow CR proton source term, by the effect of spatial diffusion, can produce a broad radial

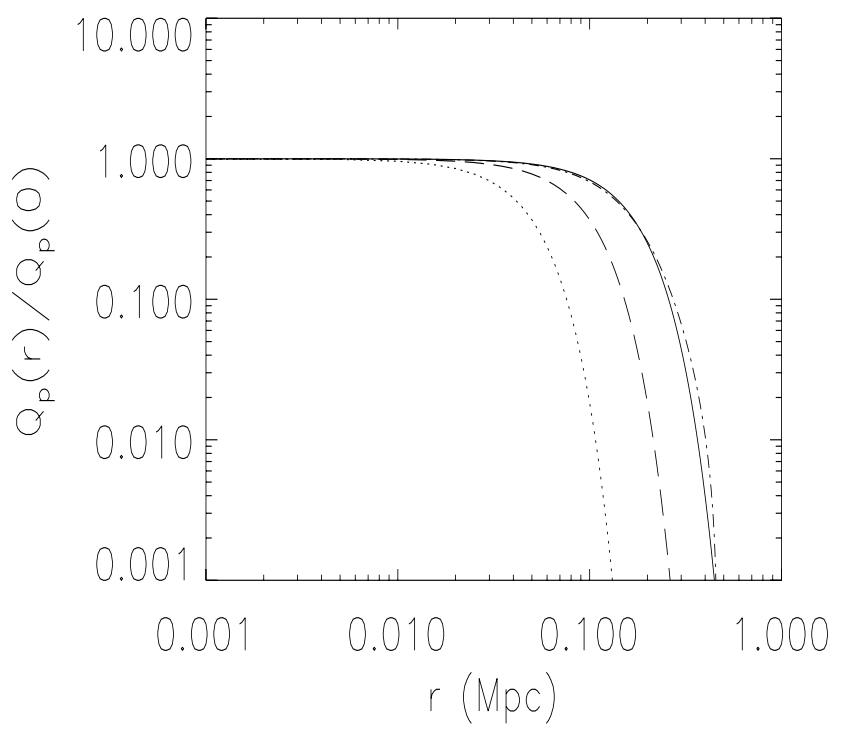

Fig. A.2. Source term (dot-dashed line) as in Fig. A.1 compared with a Gaussian shape as in Eq. (A.14) with $r_{\mathrm{s}}=0.17 \mathrm{Mpc}$ (solid line), $r_{\mathrm{s}}=0.10 \mathrm{Mpc}$ (dashed line), and $r_{\mathrm{s}}=0.05 \mathrm{Mpc}$ (dotted line).

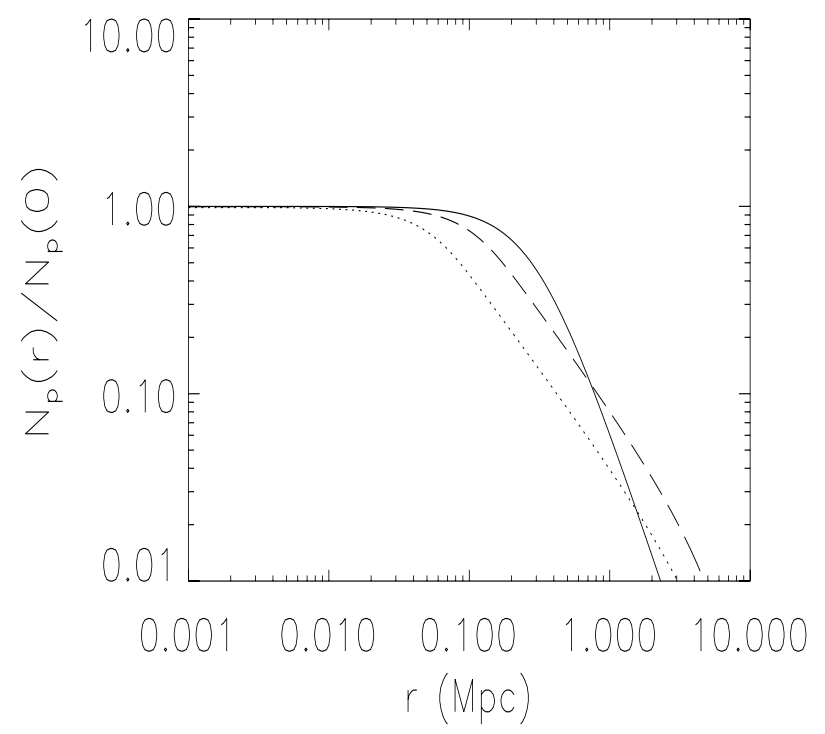

Fig. A.3. Equilibrium distribution of the protons produced by a Gaussian source term as in Eq. (A.14) with $r_{\mathrm{s}}=0.17 \mathrm{Mpc}$ (solid line), $r_{\mathrm{s}}=0.10 \mathrm{Mpc}$ (dashed line), and $r_{\mathrm{s}}=0.05 \mathrm{Mpc}$ (dotted line).

equilibrium distribution of the CR protons which is very close to the thermal IC gas distribution.

\section{References}

Allen, S. W., Schmidt, R. W., \& Fabian, A. C. 2001, MNRAS, 328, L37 Allen, S. W., Schmidt, R. W., \& Fabian, A. C. 2002, MNRAS, 335, 256 Arnaud, M. 2005 [arXiv: astro-ph/0508159]

Augusto, P., Edge, A. C., \& Chandler, C. J. 2006, MNRAS, 367, 366

Bacchi, M., Feretti, L., Giovannini, G., \& Govoni, F. 2003, A\&A, 400, 465

Bayer-Kim, C. M., Crawford, C. S., Allen, S. W., Edge, A. C., \& Fabian, A. C. 2002, MNRAS, 337, 938

Bird, J. C., Martini, P., \& Kaiser, C. 2007, ApJ, in press [arXiv:0709. 2167] Blanton, E. L., Sarazin, C. L., McNamara, B. R., \& Wise, M. W. 2001, ApJ, 558, L15

Blanton, E. L., Sarazin, C. L., \& McNamara, B. R. 2003, ApJ, 585, 227

Blasi, P., \& Colafrancesco, S. 1999, Astropart. Phys., 12, 169

Böhringer, H., \& Morfill, G. E. 1988, ApJ, 330, 609

Böhringer, H., Belsole, E., Kennea, J., et al. 2001, A\&A, 365, L181 
Böhringer, H., Matsushita, K., Churazov, E., Ikebe, Y., \& Chen, Y. 2002, A\&A, 382,804

Bregman, J. N. 2004, Proceedings of The Riddle of Cooling Flows in Galaxies and Clusters of Galaxies, held in Charlottesville, VA, May 31 - June 4, 2003, ed. T. Reiprich, J. Kempner, \& N. Soker, 327

Briel, U. G., Henry, J. P., \& Böhringer, H. 1992, A\&A, 259, L31

Brüggen, M., \& Kaiser, C. R. 2002, Nature, 418, 301

Caffi, S., Gastaldello, F., Ghizzardi, S., \& Molendi, S. 2004, Outskirts of Galaxy Clusters: Intense Life in the Suburbs, ed. A. Diaferio, IAU Coll., 195, 137

Colafrancesco, S. 2007, New Astron. Rev., 51, 394

Colafrancesco, S., \& Blasi, P. 1998, Astropart. Phys., 9, 227

Colafrancesco, S., Marchegiani, P., \& Palladino, E. 2003, A\&A, 397, 27

Colafrancesco, S., Dar, A., \& De Rújula, A. 2004, A\&A, 413, 441

Colafrancesco, S., Marchegiani, P., \& Perola, G. C. 2005, A\&A, 443, 1

Churazov, E., Sunyaev, R., Forman, W., \& Böhringer, H. 2002, MNRAS, 332, 729

Churazov, E., Forman, W., Jones, C., \& Böhringer, H. 2003, ApJ, 590, 225

Dar, A., \& De Rújula, A. 2006 [arXiv:hep-ph/0611369]

David, L. P., Nulsen, P. E. J., McNamara, B. R., et al. 2001, ApJ, 557, 546

De Rújula, A. 2004 [arXiv: astro-ph/0411763]

Donahue, M., Voit, G. M., O’Dea, C. P., et al. 2005, ApJ, 630, L13

Dwarakanath, K. S., \& Nath, B. B. 2006, ApJ, 653, L9

Elbaz, D., Arnaud, M., \& Böhringer, H. 1995, A\&A, 293, 337

Fabian, A. C. 2000, AAS Meeting, 196, \#34.13

Fabian, A. C. 2004, AIPC, 703, 337

Fabian, A. C., \& Sanders, J. S. 2006, Proceedings of Heating vs. Cooling in Galaxies and Clusters of Galaxies, August 2006, Garching [arXiv:astro-ph/0612426]

Fabian, A. C., Sanders, J. S., Taylor, G. B., et al. 2006, MNRAS, 366, 417

Ferrando, P., Goldwurm, A., Laurent, P., et al. 2005 [arXiv:astro-ph/0508674]

Foote, E. A., \& Kulsrud, R. M. 1979, ApJ, 233, 302

Fujita, Y., \& Suzuki, T. K. 2006 [arXiv: astro-ph/0610604]

Furlanetto, S. R., \& Loeb, A. 2002, ApJ, 572, 796

Gitti, M., Ferrari, C., Domainko, W., et al. 2007, A\&A, 470, L25

Govoni, F., \& Feretti, L. 2004, International Journal of Modern Physics D, 13, 1549

Guo, F., \& Oh, S. P. 2007 [arXiv: 0706. 1274]

Heinz, S., Brüggen, M., Young, A., \& Levesque, E. 2006, MNRAS, 373, L65

Hillas, A. M. 2006 [arXiv:astro-ph/0607109]

Johnstone, R. M., Allen, S. W., Fabian, A. C., \& Sanders, J. S. 2002, MNRAS, 336, 299

Kaastra, J. S., Ferrigno, C., Tamura, T., et al. 2001, A\&A, 365, L99

Kahn, S. M., Peterson, J. R., Paerels, F. B. S., et al. 2002

[arXiv:astro-ph/0210665]
Kempner, J. C., \& Sarazin, C. L. 2000, ApJ, 530, 282

Lewis, A. D., Stocke, J. T., \& Buote, D. A. 2002, ApJ, 573, L13

Loewenstein, M., Zweibel, E. G., \& Begelman, M. C. 1991, ApJ, 377, 392

Mannheim, K., \& Schlickeiser, R. 1994, A\&A, 286, 983

Marchegiani, P., Perola, G. C., \& Colafrancesco, S. 2007, A\&A, 465, 41

Matsushita, K., Belsole, E., Finoguenov, A., \& Bohringer, H. 2002, A\&A, 386, 77

McCarthy, I., Babul, A., Bower, R. G., \& Balogh, M. L. 2007, [arXiv:0706.2768]

McNamara, B. R. 1997, in Galactic and Cluster Cooling Flows, ed. N. Soker (San Francisco: PASP), 109

McNamara, B. R., Wise, M. W., David, L. P., Nulsen, P. E. J., \& Sarazin, C. L. 2000, A\&AS, 32.13211

Miniati, F., Ryu, D., Kang, H., \& Jones, T. W. 2001a, ApJ, 559, 59

Miniati, F., Jones, T. W., Kang, H., \& Ryu, D. 2001b, ApJ, 562, 233

Molendi, S., \& Pizzolato, F. 2001, ApJ, 560, 194

Nevalainen, J., Oosterbroek, T., Bonamente, M., \& Colafrancesco, S. 2004, ApJ, 608,166

Peterson, J. R., Paerels, F. B. S., Kaastra, J. S., et al. 2001, A\&A, 365, L104

Peterson, J. R., Ferrigno, C., Kaastra, J. S., et al. 2002 [arXiv: astro-ph/0202108]

Peterson, J. R., Kahn, S. M., Paerels, F. B. S., et al. 2003, ApJ, 590, 207

Pfrommer, C., \& Enßlin, T. A. 2004, A\&A, 413, 17

Pfrommer, C., Enßlin, T. A., \& Springel, V. 2007 [arXiv: 0707. 1707]

Piffaretti, R., Jetzer, Ph., Kaastra, J. S., \& Tamura, T. 2005, A\&A, 433, 101

Reimer, O., Pohl, M., Sreekumar, P., \& Mattox, J. R. 2003, ApJ, 588, 155

Rephaeli, Y. 1987, MNRAS, 225, 851

Rephaeli, Y., \& Silk, J. 1995, ApJ, 442, 91

Reynolds, C. S., McKernan, B., Fabian, A. C., et al. 2005, MNRAS, 357, 242

Ritz, S. 2007, The First GLAST Symposium, AIPC, 921, 3

Ruszkowski, M., \& Begelman, M. 2002, ApJ, 581, 223

Ruszkowski, M., Brüggen, M., \& Begelman, M. C. 2004, ApJ, 611, 158

Ryu, D., Kang, H., Hallman, E., \& Jones, T. W. 2003, ApJ, 593, 599

Sanders, J. C., \& Fabian, A. C. 2007 [arXiv:0705. 2712]

Sijacki, D., \& Springel, V. 2006, MNRAS, 371, 1025

Skilling, J. 1971, ApJ, 170, 265

Tamura, T., Kaastra, J. S., Peterson, J. R., et al. 2001, A\&A, 365, L87

Vernaleo, J. C., \& Reynolds, C. S. 2005, A\&AS, 207, 5503

Vernaleo, J. C., \& Reynolds, C. S. 2006, ApJ, 645, 83

Vernaleo, J. C., \& Reynolds, C. S. 2007 [arXiv: 0708.4015]

Vikhlinin, A., Markevitch, M., Murray, S. S., Jones, C., Forman, W., \& Van Speybroeck, L. 2005, ApJ, 628, 655

Voit, G. M., \& Donahue, M. 2005, ApJ, 634, 955 